\title{
EXERCISE INTENSITY AND PACING STRATEGY OF A 5-KM INDOOR RACE WALK DURING A WORLD RECORD ATTEMPT: A CASE STUDY
}

Tutor

Chiar.mo Prof. Antonio LA TORRE

Coordinatore del Corso di Dottorato

$\overline{\text { Chiar.mo Prof. Livio LUZI }}$

Tesi di Dottorato di Ricerca

Gianluca VERNILLO

Matricola: R08032

Triennio Accademico

$2009 / 2012$ 


\section{SOMMARIO}

SOMMARIO

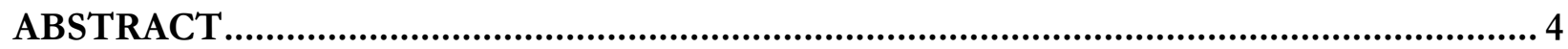

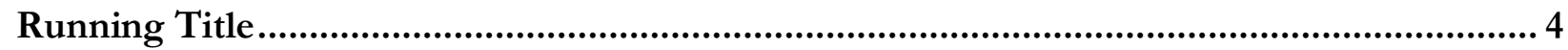

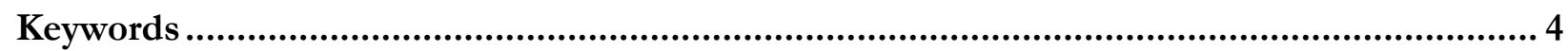

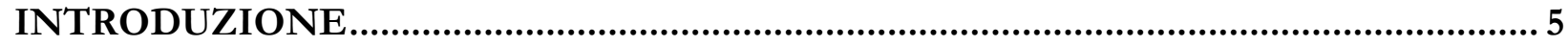

L'origine del Cammino: Un Percorso Lungo 6 Milioni di Anni .......................................... 6

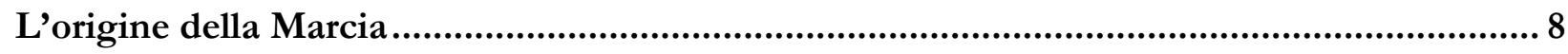

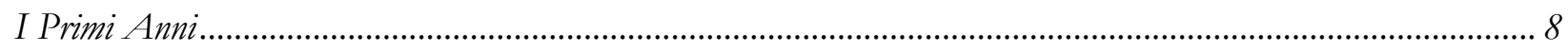

L'invasione delle Scommesse Clandestine ........................................................................................................ 8

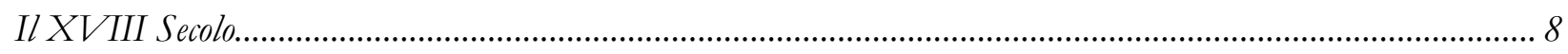

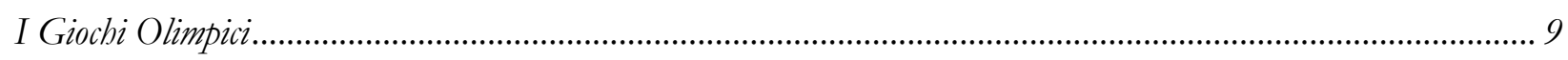

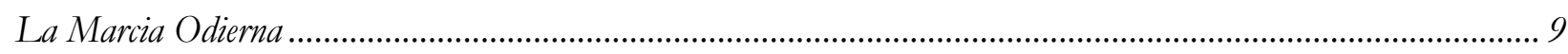

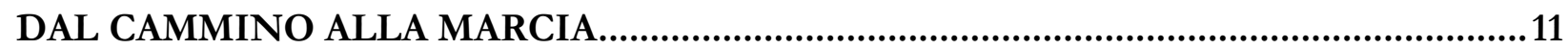

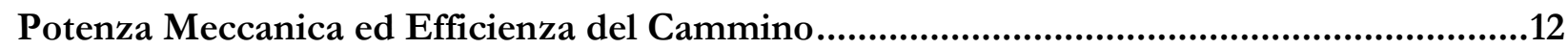

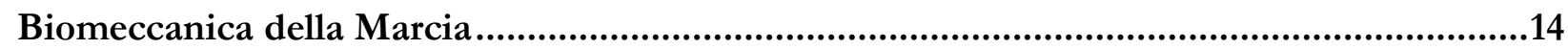

INTENSITÀ DELL'ESERCIZIO E CARICO ALLENANTE .........................................16

La Valutazione del Carico di Allenamento .........................................................................18

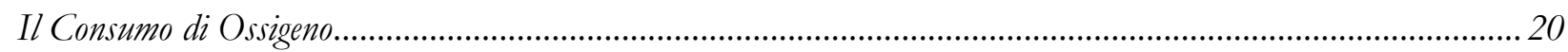

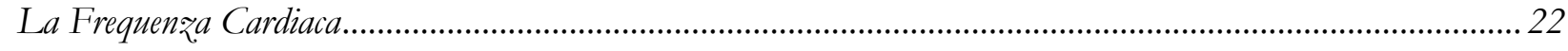

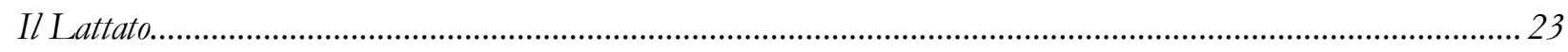

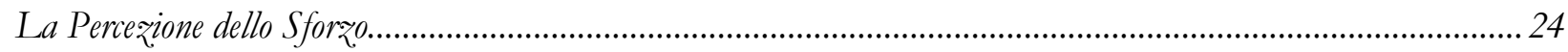

Determinazione del Carico Allenante .................................................................................... 26

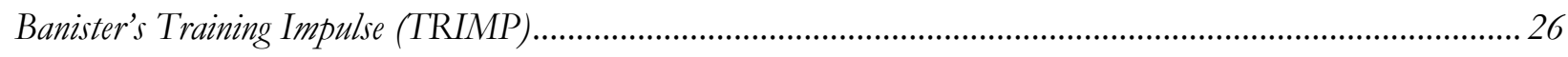

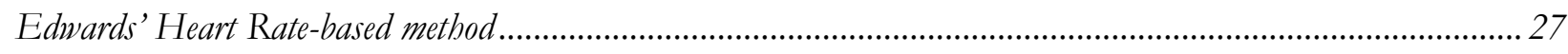

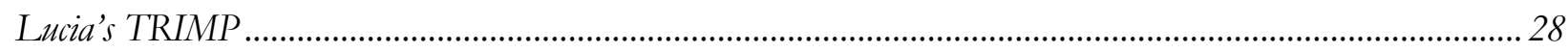

Tempo passato in zone d'intensità delimitate dalla frequenza cardiaca di soglia lattacida o ventilatoria ................... 29

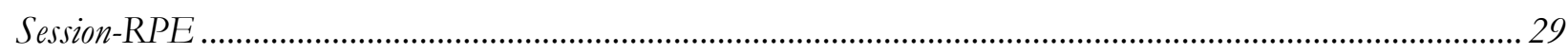

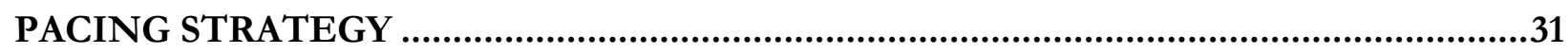

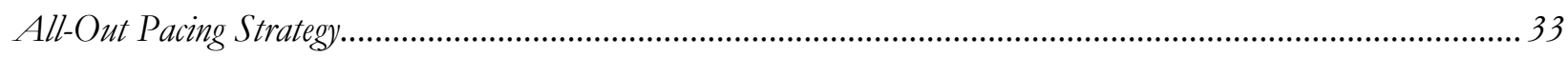




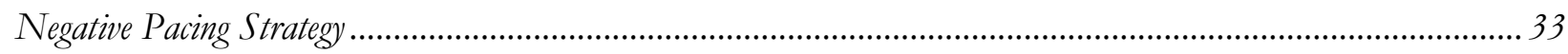

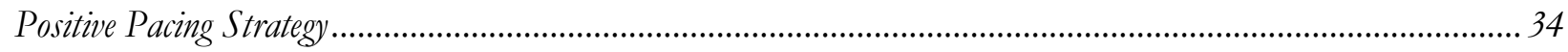

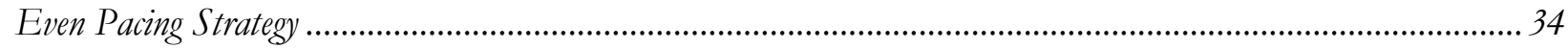

Parabolic-Shaped Pacing Strategy ........................................................................................................... 35

Variable Pacing Strategy .............................................................................................................................. 35

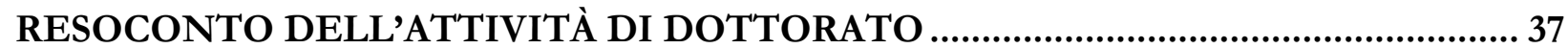

Physical Activity Intensity, Heart Rate, Blood Lactate Concentration and RPE During 5-Km Men's and 3-Km

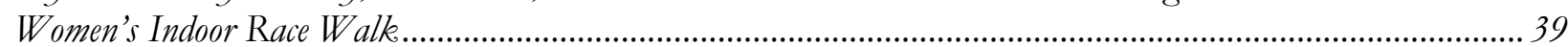

The Training for the 20-Km RW. What Is Changed and What Might Change? ................................................ 41

Pacing Strategy During 50-Km Race Walking Events .............................................................................. 42

Technical Warnings-Related Effects on the Performance During a 50-Km Race Walk Competition...................... 44

Quantifying the Training Load During a 10000-m Race Walk........................................................................ 46

Variations of Perceived Exertion in Relation to Heart Rate Responses in an International Level Race Walkers

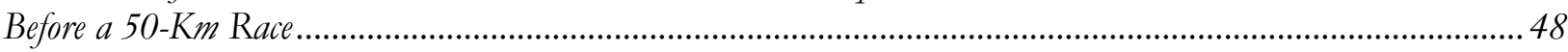

An Observational Study on the Perceptive and Physiological Variables During a 10,000-m Race Walking

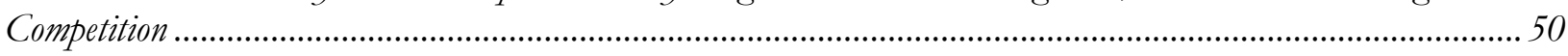

Cardiovascular Responses During an Indoor Race Walking Competition ........................................................ 51

EXERCISE INTENSITY AND PACING STRATEGY OF A 5-KM INDOOR RACE WALK DURING A WORLD RECORD ATTEMPT: A CASE STUDY.......................... 52

INTRODUCTION

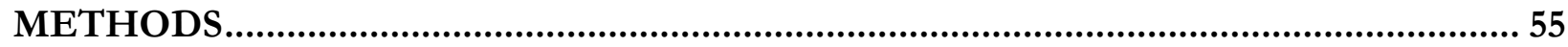

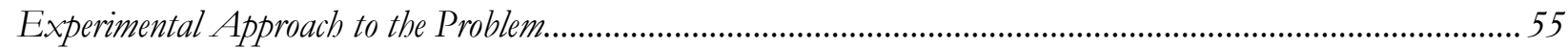

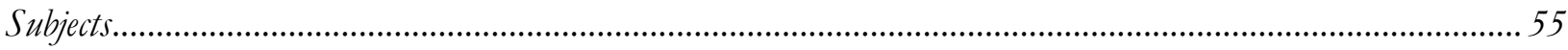

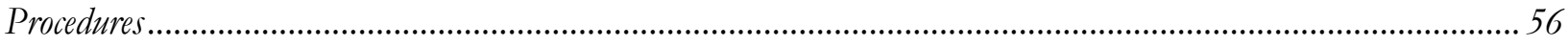

Statistical Analyses............................................................................................................................. 57

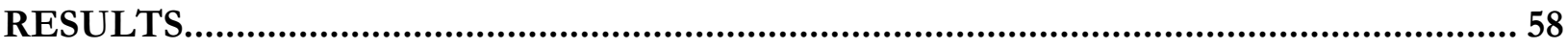

DISCUSSION

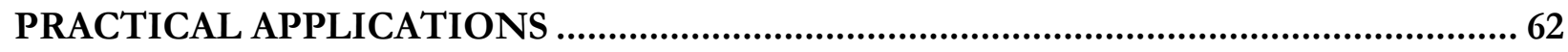

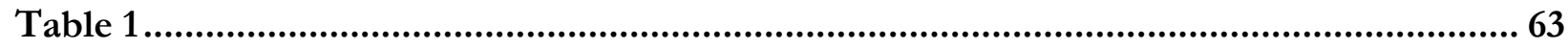

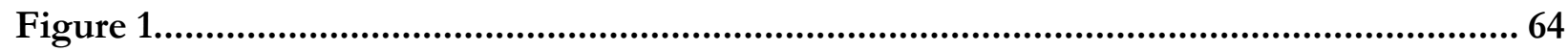

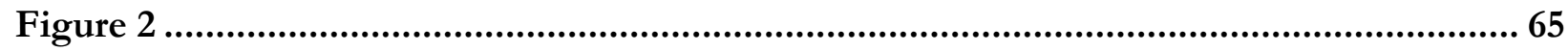

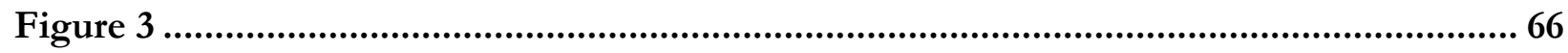

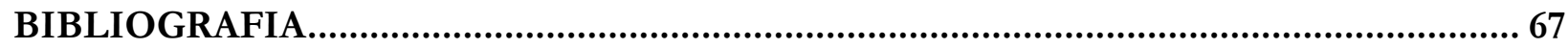




\section{ABSTRACT}

Exercise intensity and pacing strategy of a 5-km indoor race walk during a world record attempt: A case study (Vernillo, Piacentini, Drake, Agnello, Fiorella \& La Torre 2011). The aim of this case study was to describe the physiological and regulatory processes, by means of heart rate $(\mathrm{HR})$ monitoring and pacing strategy, in a top-level race walker (age: 32 years; height: $1.76 \mathrm{~m}$; body mass: $62 \mathrm{~kg}$; training volume: $130-150 \mathrm{~km} \cdot \mathrm{wk}^{-1}$ ) who was focused on the attainment of the 5$\mathrm{km}$ indoor race walk (RW) World Record. The HRmean was $185614.9 \mathrm{~b} \cdot \mathrm{min}^{-1}$, with an HRmean/HRmax ratio of 0.96 . Almost the whole race (91.8\%) was performed to an intensity $\geq 90 \%$ of the HRmax; lower intensity work was negligible (8.1\%). The race profile was a reverse J-shaped pacing curve; in fact, the athlete completed the first $1,000 \mathrm{~m}$ in the fastest time, slowing during the middle 3,000 $\mathrm{m}$, and increasing the speed during the final 1,000 $\mathrm{m}$ of the race. Despite the attempt failed (the athlete performed only the 2009 World leading performance, 18 minutes 23 seconds 47 tenths), these data suggest that a more linear strain distribution for the entire performance would be optimal instead of a fast-start strategy, which leads to a drastic decrement of the walking velocity. Moreover, this study supports the use of HR monitoring combined with the regulation of the effort to understand the physiological and regulatory processes during an indoor RW event.

\section{Running Title}

Intensità dell'Esercizio e Distribuzione dello Sforzo nella Marcia

\section{Keywords}

athlete, endurance, perceived exertion, exercise performance, heart rate 


\section{INTRODUZIONE}




\section{L'origine del Cammino: Un Percorso Lungo 6 Milioni di Anni}

Una delle più frequenti è vecchie domande nel campo della paleo-antropologia, che continua ad intrigare l'opinione scientifica attuale nonché quella pubblica, riguarda quando e, soprattutto, perché i più antichi membri del genere umano cominciarono a camminare su due arti. Nel corso degli anni numerose ipotesi sono state formulate riguardo gli agenti responsabili dell'origine del bipedismo, molte delle quali dipendono dalla forma del comportamento motorio che lo precedettero e che hanno influenzato il bipedismo stesso e da cui quest'ultimo si è evoluto. Brevemente, si riconoscono 5 grandi filoni di pensiero (Richmond, Begun \& Strait 2001):

1. Arboreal quadruped ancestor. In cui il bipedismo si evolse da un antenato abituato a vivere sui rami, con una deambulazione prona in quadrupedismo proprio come la maggior parte degli antropoidi viventi.

2. Terrestrial quadruped incesto. In cui il bipedismo si evolse da un antenato che si adattò a qualche forma di quadrupedia terrestre.

3. Hylobatian incesto. In cui il bipedismo si evolse da un antenato poco incline al brachiatismo (i.e., forma di movimento che prevede il passaggio da ramo a ramo mediante l'oscillazione degli arti anteriori, tipico di molti gibboni) (Fleagle 1976).

4. Climbing (antipronograde) incesto. In cui il bipedismo si evolse da un antenato che principalmente si era adattato alla locomozione arborea che implicava una mobilità considerevole degli arti anteriori e posteriori, una postura sospensoria e l'uso di molteplici supporti spesso di tipo verticale.

5. Knuckle-walking incesto. In cui il bipedismo si evolse da un antenato che utilizzava come componente principale di locomozione bipede la camminata sulle nocche.

Indipendentemente da quale ipotesi ad oggi risulti essere la più consistente da un punto di vista scientifico, è opinione della comunità internazionale che i primi ominidi (i.e., primati che 
abitualmente usavano una deambulazione bipodalica in posizione eretta) sono apparsi e si sono evoluti in Africa tra i 5 ed i 6 milioni di anni fa (Fleagle 1979; Senut 2002; Ward, Leakey, Brown, Brown, Harris \& Walker 1999). Questi erano membri del genere Australopithecus ed erano caratterizzati da una corporatura ridotta (se paragonata all'essere umano moderno) ed i loro scheletri contenevano un mosaico di caratteristiche differenti (e.g., articolazioni relativamente mobili, arti superiori ed inferiori relativamente lunghi e corti, rispettivamente etc.) (Leakey, Feibel, McDougall \& Walker 1995; Lovejoy 1988; Stern \& Susman 1983; Susman \& Stern 1982; Ward et al., 1999). Le loro caratteristiche di deambulazione si differenziavano da quelle della stragrande maggioranza dei mammiferi a causa delle diverse richieste meccaniche che gravavano sugli arti durante la locomozione, comportamento che si pensa sia stato decisivo nell'origine dei primati 55 milioni di anni fa e che differenzi a livello funzionale i bipedi dai quadrupedi (Fleagle 1979; Schmitt 2003a, 2003b). Di conseguenza, sin dagli studi pioneristici dell'anatomo Britannico Herbert Elftman (Elftman 1951, 1938; Elftman \& Manter 1935), una delle caratteristiche principali che separa l'essere umano dagli altri primati è l'abituale uso della deambulazione bipede, ovvero del cammino (Schmitt 2003b). 


\section{L'origine della Marcia}

La naturale evoluzione del processo di selezione che portò i primati di 55 milioni di anni fa ad usare una deambulazione bipodalica è racchiusa nella natura stessa dell'essere umano. Infatti, non ci volle 'molto' per trasformare una delle attività più frequenti e praticate in una competizione.

\section{Primi Anni}

A conferma di ciò, le prime tracce di competizioni di marcia si ritiene risalgano addirittura al 2500 a.C., grazie agli Egizi che con la loro forma di scrittura (i.e., i geroglifici) testimoniarono il primo documento di una competizione di cammino. Evidenze simili furono poi riscontrate nel corso della storia anche nell'antica Grecia. Tuttavia, queste prime testimonianze mostravano una mancanza di regole ben definite; il che permetteva agli atleti in gara anche di alternare fasi di corsa a fasi di cammino. Sono comunque testimonianze importanti per datare l'origine di questa disciplina per come la conosciamo noi oggi.

\section{L'invasione delle Scommesse Clandestine}

Una delle evoluzioni non propriamente naturali di questa disciplina si ebbe nel XIV secolo quando fiumi di scommesse clandestine cominciarono a gravare sulle imprese di alcuni atleti che marciando compivano distanze ai più proibitive. Un famoso esempio risale in Inghilterra nel 1589 quando un nobiluomo inglese, Sir Robert Carey, scommise che poteva marciare ininterrottamente per 300 miglia (i.e., 482.8 chilometri). Vinta la scommessa gettò le basi per un'impresa ancor più sensazionale, che portò a termine nel 1608 quando marciò per 2000 miglia (i.e., 3218.7 chilometri) attraverso l'Europa in circa 41 giorni.

\section{XVIII Secolo}

Sebbene le imprese di compiere lunghe distanze da parte di marciatori catturassero certamente l'immaginario pubblico, non ci volle molte prima di trasformare queste 'epiche imprese' in 
competizioni ufficiali, ovviamente con delle varianti. I nobiluomini non si battevano che per la gloria, quindi iscrivevano alle gare dei 'gladiatori' che portavano a termine le loro scommesse. I primi marciatori del XVII e XVIII secolo altro non erano che i valletti dei nobiluomini, che come mezzo di allenamento usavano camminare in parte alla carrozza del loro signore e, talvolta, precedevano il signore stesso per sbrigare i suoi affari in città prima che egli stesso arrivasse.

Tuttavia si iniziò a parlare di questa disciplina come di uno sport solo alla metà del XVIII secolo. La seconda metà dello stesso secolo vide, appunto, i primi marciatori darsi battaglia tra di loro e contro il tempo sia per gloria che per la ricchezza.

\section{Giochi Olimpici}

Nonostante agli inizi del XX secolo la popolarità della marcia fosse in declino, essa riuscì a riemergere seppur di poco quando fu considerata disciplina Olimpica. Molte persone ritengono che questo evento sia da far risalire al 1908 (Giochi Olimpici di Atene, Grecia); tuttavia attualmente si ritiene debba essere leggermente anticipato. Nel 1904 (Giochi Olimpici di St. Louis, Stati Uniti), la marcia fece la sua prima comparsa nello scenario Olimpico come disciplina inserita nel decathlon (880-yard, 0.8 chilometri).

\section{La Marcia Odierna}

Tutto il passaggio storico brevemente descritto precedentemente portò alla creazione dell'attuale marcia agonistica (in inglese Race Walking o Race Walk). La marcia odierna è governata principalmente da due commi delle regola 230 definiti dell'Associaz̧one Internazionale delle Federazioni di Atletica (i.e., International Association of Athletics Federations - IAAF) (IAAF 2009):

1. La marcia è una progressione di passi in cui il marciatore deve essere sempre a contatto col terreno in modo tale che nessuna perdita di contatto con esso sia visibile ad occhio nudo. 
2. La gamba avanzante deve essere completamente distesa (i.e., cioè non flessa al ginocchio) dal primo momento di presa di contatto col terreno fino al passaggio sulla proiezione verticale del centro di massa dell'arto inferiore stesso.

L'andatura che viene eseguita durante l'azione di marcia è, dunque, in funzione delle regole stesse che governano questa disciplina, che generano un modello di movimento con delle caratteristiche biomeccaniche assolutamente uniche (Cairns, Burdett, Pisciotta \& Simon 1986). 


\section{DAL CAMMINO ALLA MARCIA}




\section{Potenza Meccanica ed Efficienza del Cammino}

Quando l'essere umano moderno deambula una delle caratteristiche principali è il 'volteggiare' sopra ai suoi arti inferiori in modo tale che il suo centro di massa si trovi nel punto più basso alla presa di contatto del tallone con la superficie, per arrivare al punto più alto al passaggio dell'arto sulla proiezione verticale del centro di massa (Cavagna, Thys \& Zamboni 1976; Lee \& Farley 1998). Quest'azione genera una curva di forza verticale al suolo con due picchi caratteristici entrambi maggiori del peso corporeo, al contrario dei primati non-umani che, durante il cammino, presentano un solo picco che generalmente è pari al peso corporeo (Crompton, Vereecke \& Thorpe 2008; Kimura 1996; Umberger \& Martin 2007).

Oltre alla presenza caratteristica di questo doppio picco, la deambulazione umana è caratterizzata da un modello di movimenti il più economico possibile. Negli corso degli anni numerosi studi hanno evidenziato come il cammino presenti una biomeccanica definita come 'pendolo inverso"1 (Bramble et al., 2004). Il costo metabolico per questo 'sistema di trasporto', come per gli altri mammiferi, è rappresentato da una 'curva U-shaped' (Minetti, Capelli, Zamparo, di Prampero \& Saibene 1995; Zarrugh \& Radcliffe 1978), in cui la velocità di deambulazione ottimale, approssimativamente 1.3 $\mathrm{m} \cdot \mathrm{s}^{-1}$, è largamente influenzata dalla lunghezza degli arti inferiori (Bramble et al., 2004) ed il suo minimo, generalmente, coincide con la lunghezza del passo preferita (Umberger et al., 2007). Con l'aumentare della velocità di cammino, oltre che ad un aumento del costo metabolico, l'essere umano sceglie di cambiare la modalità di deambulazione, passando dal cammino alla corsa (i.e., punto di transizione) che generalmente di assesta tra i $2.3 \mathrm{ed} \mathrm{i} 2.5 \mathrm{~m} \cdot \mathrm{s}^{-1}$. Questo perché con l'aumento della

${ }^{1} \mathrm{Il}$ pendolo inverso è un pendolo in cui la massa principale si trova sopra il fulcro di rotazione; ovvero il centro di massa 'volteggia' sopra l'arto inferiore pressoché esteso durante la fase di appoggio nel cammino (Bramble \& Lieberman 2004). 
velocità la corsa diventa il 'sistema di trasporto' più economico grazie all'intervento del meccanismo di ritorno elastico che modifica l'energia cinetica e potenziale in maniera considerevole (Bramble et al., 2004). 


\section{Biomeccanica della Marcia}

Da un punto di vista biomeccanico la marcia possiede delle proprietà sia di tipo cinematico che di tipo cinetico uniche (Cairns et al., 1986). Ciò è dovuto principalmente al lavoro svolto dagli arti inferiori durante l'azione tecnica che permette un miglioramento dell'efficienza meccanica (Cavagna \& Franzetti 1981; Murray, Guten, Mollinger \& Gardner 1983). Nello specifico quanto sopra riportato è possibile mediante un aumento (i) della flessione plantare, e (ii) della flessione dorsale dell'arto inferiore posteriore ed anteriore completamento teso, rispettivamente. Questo genera un movimento ellittico del bacino che consente di ridurre al minimo la fluttuazione del centro di massa (soprattutto nella sua componente verticale) e, di conseguenza, migliorare il costo energetico stesso (Ziv \& Rotstein 2009). Infatti è stato osservato come marciatori di alto livello raggiungono anche velocità doppie rispetto alla massima velocità di cammino (i.e., $2 \mathrm{~m} \cdot \mathrm{s}^{-1}$ ca.), ma con un aumento molto meno considerevole nella spesa energetica (Menier \& Pugh 1968). Di conseguenza, a parità di velocità di spostamento la marcia sembrerebbe avere un costo inferiore rispetto al cammino principalmente imputabile a due fattori (Cavagna et al., 1981):

1. Un minor lavoro meccanico esercitato per lo spostamento.

2. Un'efficienza maggiore del lavoro positivo.

A questo va aggiunto inoltre come rispetto al cammino od alla corsa la marcia possiede anche altre caratteristiche intrinseche proprie. Infatti, è stato osservato come, oltre ad una più marcata flessione plantare e dorsale a livello della caviglia ed uno movimento specifico del bacino, nella marcia si assiste anche ad (Cairns et al., 1986):

1. Una massima estensione a livello del ginocchio.

2. Un aumento del momento di picco esterno durante l'iperestensione del ginocchio.

3. Un aumento delle forze generate al suolo dal piede. 
4. Un aumento del momento di picco della flessione plantare.

Le sopra citate caratteristiche biomeccaniche fanno sì che gli atleti riescano a 'marciare' a più alte velocità senza per questo dover eseguire un'azione di corsa ${ }^{2}$. Infatti, è stato osservato come nei marciatori il punto di transizione preferito ed il punto di transizione ottimale da un punto di vista energetico siano maggiori e nettamente più ravvicinati rispetto a quanto si abbia nel cammino e nella corsa (Ziv et al., 2009).

Tuttavia, nonostante le sopracitate caratteristiche intrinseche, anche la marcia risponde a determinati patterns locomotori che sono propri anche del cammino e della corsa (Cavanagh \& Williams 1982). Infatti, è stato osservato come anche in marciatori di alto livello il costo energetico peggiorava a seconda che la lunghezza del passo (ad una determinata velocità di spostamento) venisse alterata sia in difetto (i.e., riducendola) od in eccesso (i.e., aumentandola) (Morgan \& Martin 1986).

${ }^{2}$ Si ricorda che il punto di transizione dal cammino alla corsa generalmente si assesta tra i 2.3 ed i $2.5 \mathrm{~m} \cdot \mathrm{s}^{-1}($ Bramble et al., 2004). 


\section{INTENSITÀ DELL'ESERCIZIO E CARICO ALLENANTE}


L'allenamento è la sistematica ripetizione di esercizi fisici e può essere descritto in base ai suoi adattamenti ed ai suoi processi. Gli adattamenti all'allenamento sono di tipo anatomico, fisiologico, biomeccanico e funzionale e sono specifici di ogni disciplina sportiva; mentre il processo essenzialmente può essere considerato come il carico di allenamento (ovvero il prodotto del volume e dell'intensità di un allenamento). Esso, quindi, può essere definito come un indice dell'impegno richiesto per eseguire una certa seduta o della fatica che da essa ne è derivata (i.e., carico di lavoro come effetto allenante). Di conseguenza, con la determinazione del carico allenante si cerca di misurare il lavoro prodotto durante l'allenamento stesso, che può essere suddiviso in (Booth \& Thomason 1991):

- esterno: è rappresentato dalla quantificazione oggettiva dei mezzi adottati nell'allenamento (km percorsi, tempo impiegato a percorrerli, etc.).

- interno: è definito come lo stimolo (stress) fisiologico che il mezzo di allenamento induce sulle proprietà strutturali e funzionali delle cellule, degli organi e degli apparati.

Dunque, gli adattamenti indotti dall'allenamento sono il risultato della ripetizione organizzata e strutturata di esercizi fisici ed ogni cambiamento è legato alla natura, intensità e durata dello sforzo dell'esercizio. Ma lo stimolo allenante, che quindi genera un adattamento, non è altro che il relativo stress fisiologico che viene imposto all'organismo dell'atleta, ovvero il carico allenante interno e non il carico allenante esterno (Viru \& Viru 2000). Questo carico allenante interno è determinato da caratteristiche individuali (e.g., patrimonio genetico ed il livello iniziale dell'allenamento) e dalla qualità, quantità e dall'organizzazione del carico allenante esterno (Impellizzeri, Rampinini \& Marcora 2005). Dunque, per poter monitorare e controllare l'allenamento è assai importante valutare sia gli adattamenti che il carico interno dell'allenamento imposto in quel momento. 


\section{La Valutazione del Carico di Allenamento}

Per carico di allenamento si intende la combinazione di tre fattori:
1. Intensità.
2. Durata.
3. Frequenza.

Di conseguenza, gli adattamenti ottimali conseguenti all'allenamento saranno appropriati se la quantità del carico allenante sarà applicata agli atleti (indipendentemente dal loro livello competitivo) in maniera appropriata (Smith 2003). Il carico di allenamento può essere classificato come i carichi di una singola seduta o, ad esempio, di un micro ciclo come segue (Viru 1995):

- Carico Eccessivo. Si intende il carico che eccede la capacità funzionale dell'organismo portando ad una sorta di over-reaching e/o over-training).

- Carico Allenante. Che risulta in uno specifico effetto allenante.

- Carico di Mantenimento. Carico sufficiente per evitare degli effetti de-allenanti.

- Carico di Recupero. Che favorisce i processi di recupero a seguito di carichi eccessivi o di allenamento.

- Carico Inadeguato. Al di sotto dell'intensità o del valore necessario a raggiungere nessun effetto allenante.

I fattori determinanti il carico interno includono (Siff \& Verkhoshansky 1999):

- La Specificità dell'Allenamento. Ovvero l'allenamento a carattere generale vs. specifico.

- Gli Stimoli Allenanti del Carico. 
- La Quantificazione dell'Allenamento. Che dovrebbe essere in relazione al livello di performance dell'atleta.

- La Durata e l'Intensità dei cicli di allenamento nel corso dell'anno.

- La Sequenza e l'Interazione degli elementi tecnico-allenanti in discipline altamente tecniche.

Ad oggi, esistono diverse metodiche utili alla determinazione sia dell'intensità di un esercizio fisico che del relativo carico allenante. Esse rappresentano degli strumenti validi, utili (ed a volte di semplice applicazione) per stimare ciò che accade, o che potrebbe accadere, all'organismo di un atleta. Solitamente questa valutazione può essere fatta o valutando la durata di uno sforzo (i.e., contesto temporale) oppure valutando l'intensità dello sforzo stesso (i.e., ambito fisiologico). Se l'utilizzo del tempo è una pratica relativamente semplice, ma che può dare un certo margine di errore in quanto si avrebbe un dato dell'andamento temporale di uno sforzo, ma in realtà ciò che succede all'organismo dell'atleta si può solo immaginare (i.e., si conoscerebbe molto bene il carico esterno, ma poco quello interno), di maggior interesse potrebbe essere la comprensione e l'utilizzo di strumenti utili per determinare l'intensità di uno sforzo attraverso misure di tipo fisiologico come (Borresen \& Lambert 2009):

1. Il Consumo di Ossigeno.

2. La Frequenza Cardiaca.

3. Il Lattato.

4. La Percezione dello Sforzo. 


\section{Il Consumo di Ossigeno}

Il consumo di ossigeno (i.e., $\dot{\mathrm{V}} \mathrm{O}_{2}$ ) è generalmente riconosciuto dalla letteratura scientifica come misura valida per determinare e prescrivere l'intensità di un esercizio. Tuttavia, questo utilizzo sembrerebbe dare delle risposte attendibili solo in presenza di esercizi a carico costante (i.e., steadystate) e non intermittenti. Questo perché (i) solo la relazione tra consumo di ossigeno ed un lavoro a carico costante risulta essere lineare (Robinson, Robinson, Hume \& Hopkins 1991) ed (ii) in presenza di un carico di lavoro al di sotto della soglia lattacida, il consumo di ossigeno cresce in maniera esponenziale fino ad un livello costante, ma quando si è in presenza di un carico sopra la suddetta soglia la sua cinetica diventa maggiormente complessa (Xu \& Rhodes 1999).

Come per altri parametri fisiologici (e.g., frequenza cardiaca) il consumo di ossigeno dovrebbe essere sempre espresso in termini relativi e mai assoluti. Esprimere un parametro in termini relativi altro non vuol dire che rapportarlo a percentuali rispetto al massimo valore del parametro stesso registrato. Quindi, nel caso del consumo d'ossigeno il dato relativo altro non è che la percentuale di lavoro rispetto al massimo consumo di ossigeno ${ }^{3}$ (i.e., $\% \dot{\mathrm{V}} \mathrm{O}_{2 \max }$ ) (Hopkins 1991). Tuttavia, è stato determinato che il massimo consumo di ossigeno è specifico per ogni tipologia di esercizio; dunque esso richiederebbe di essere determinato prima che l'esercizio stesso possa essere prescritto o quantificato usando i valori relativi (Kohrt, Morgan, Bates \& Skinner 1987). A questo proposito, il

${ }^{3}$ Il massimo consumo di ossigeno è definito come il più alto tasso al quale l'ossigeno può essere estratto ed utilizzato dal'organismo durante un esercizio (Bassett \& Howley 2000).

È generalmente riconosciuto come la più valida misura del limite funzionale del sistema cardiovascolare ed solitamente utilizzato come indice per determinare la fitness cardiorespiratoria (Howley, Bassett \& Welch 1995; Rowell 1974). 
consumo di ossigeno di riserva (i.e., $\dot{\mathrm{V}} \mathrm{O}_{2} \mathrm{R}$, equazione 1) è stato suggerito come un valore più accurato col quale prescrivere l'intensità di un esercizio rispetto a $\% \dot{\mathrm{V}} \mathrm{O}_{2 \max }$ :

$$
\% \dot{\mathrm{V}} \mathrm{O}_{2} \mathrm{R}=\frac{\dot{\mathrm{V}} \mathrm{O}_{2 \mathrm{exp}} \cdot \dot{\mathrm{V}} \mathrm{O}_{2 \mathrm{rent}} \cdot 100}{\dot{\mathrm{V}} \mathrm{O}_{2 \max } \cdot \dot{\mathrm{V}} \mathrm{O}_{2 \mathrm{rest}}} \quad \text { (Equazione 1) (Borresen et al., 2009) }
$$

È stato dimostrato sia nel ciclismo che nella corsa che mentre calcolare l'intensità dell'esercizio mediante l'utilizzo del consumo di ossigeno di riserva e della frequenza cardiaca di riserva (paragrafo successivo) dia risultati simili, le intensità calcolate usando il massimo consumo di ossigeno differiscono (Swain \& Leutholtz 1997; Swain, Leutholtz, King, Haas \& Branch 1998). È stato osservato, inoltre, che la frequenza cardiaca ed i markers plasmatici di stress dovuti ad un esercizio (e.g., lattato, ioni ammonio etc.) al 70\% $\dot{\mathrm{V}} \mathrm{O}_{\text {2peak }}$ differivano tra soggetti allenati e non (Baldwin, Snow \& Febbraio 2000). Questo potrebbe supportare l'ipotesi che usare percentuali del picco di consumo di ossigeno non necessariamente produce le stesse risposte fisiologiche in differenti popolazioni di soggetti. Inoltre, è stato osservato che le cinetiche del consumo di ossigeno all'inizio dell'esercizio possono differire a seguito:

1. Del livello di allenamento.

2. Dell'età.

3. Del livello di sedentarietà.

Di conseguenza, l'utilizzo del consumo di ossigeno può essere inappropriato per prescrivere intensità di esercizio relative (Baldwin et al., 2000; Skinner, Wilmore, Krasnoff, Jaskolski, Jaskolska, Gagnon et al., 2000). 


\section{La Frequenza Cardiaca}

L'utilizzo della frequenza cardiaca (HR) per descrivere e determinare l'intensità di un esercizio fisico si basa sulla relazione lineare tra la frequenza cardiaca ed il massimo consumo di ossigeno determinata su un largo range di intensità sub-massimali in condizioni di steady-state (Åstrand \& Rodahl 1986). Di conseguenza la frequenza cardiaca risulterebbe essere un indice attendibile per determinare lo stress fisiologico occorso all'atleta durante un qualsiasi tipo di sforzo (Impellizzeri, Rampinini, Coutts, Sassi \& Marcora 2004; Reilly 1997). Dunque, attraverso l'utilizzo della frequenza cardiaca è possibile determinare l'intensità di un esercizio, ma, come per il consumo di ossigeno, è di estrema importanza non riferirsi mai al valore assoluto (i.e., al dato in sé), ma sempre considerare la frequenza cardiaca:

1. In percentuale della massima frequenza cardiaca.

2. In percentuale rispetto alle soglie (ventilatorie e/o lattacide).

3. In percentuale della riserva di frequenza cardiaca (HRR, equazione 2):

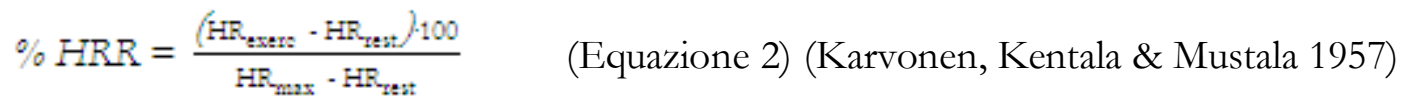

Questo perché nonostante le misure relative sembrerebbero fornire più informazioni, differenze sostanziali sia intra- che inter-individuali possono esistere nel modo con cui i soggetti rispondono ai diversi tipi di esercizio (Borresen et al., 2009).

Nonostante i cardiofrequenzimetri odierni misurano in maniera accurata la frequenza cardiaca durante un'attività fisica, molti fattori possono influenzare la relazione esistente tra il carico di lavoro e la frequenza stessa. Tuttavia se i fattori che potenzialmente influenzano la frequenza cardiaca come 
la variabilità giornaliera, il livello di allenamento, le condizioni ambientali, la durata dell'esercizio, lo stato d'idratazione, l'altitudine e i farmaci sono controllati, l'accuratezza con la quale la frequenza cardiaca può essere usata come marker dell'intensità dell'esercizio aumenta (Achten \& Jeukendrup 2003; Lambert, Mbamba \& St Clair Gibson 1998; Robinson et al., 1991).

\section{Lattato}

Le prime osservazioni di un aumento repentino del lattato prodotto dal nostro organismo durante un esercizio incrementale risalgono addirittura all'inizio del secolo scorso (Barcroft \& Margaria 1932; Christiansen, Douglas \& Haldane 1914; Latham 1908; Owles 1930). Negli ultimi anni la valutazione della concentrazione di lattato ematico si è notevolmente semplificata grazie allo sviluppo di strumenti di analisi portatili che richiedono il campionamento di una sola goccia di sangue dal polpastrello del dito oppure dal lobo dell'orecchio. Grazie a questo miglioramento nella strumentazione, particolare attenzione è stata riposta nella valutazione della soglia lattacida, definita come l'intensità di un esercizio ad un livello di lattato ematico fisso o massimale (Cairns 2006). Tuttavia, il dibattito su quanto esso possa essere 'amico o nemico' (Cairns 2006) della performance è ampio. Se consideriamo che l'accumulo di lattato ematico durante esercizi whole-body può essere temporaneamente associato con un'influenza negativa sulla performance a causa dell'associazione di questo accumulo con altri parametri metabolici (Green 1997); potremmo considerarlo allo stesso modo come un buon marker per determinare l'intensità dell'esercizio ed il conseguente adattamento all'allenamento (Cairns 2006). In quest'ottica, il tentativo che si potrebbe fare praticamente non sarebbe solamente quello di determinare un valore di lattato durante uno sforzo (in quanto risulterebbe un dato fine a sé stesso, di poca importanza), ma sarebbe più utile, a nostro avviso, determinare una serie di valori di lattato ematico (e.g., con una serie di prelievi durante uno sforzo o al termine), di modo da aver un'idea più precisa su quella che potrebbe essere la sua cinetica (Oosthuyse \& Carter 1999) e di conseguenza il training status di un atleta e gli adattamenti nel tempo. 


\section{La Percezione dello Sforzo}

Il concetto di percezione dello sforzo ed i metodi per la sua quantificazione nascono negli anni ‘50 del secolo scorso ad opera di Borg e Dahlström. Dato interessante è che, dal 1962 ad oggi, sono oltre 2200 gli studi sulla percezione dello sforzo che, oltre all'ambito delle sport sciences, sono stati condotti in numerosi campi (e.g.):

- Per il controllo del diabete in adolescenti (Hanson \& Pichert 1986).

- Per il controllo delle posture da lavoro (Wangenheim, Carlsoo, Nordgren \& Linroth 1986).

- Insufficienza cardiaca (Green, Watts, Maiorana \& O'Driscoll 2001).

La percezione dello sforzo (in inglese Rating of Perceived Exertion - RPE) è stata definita come la capacità di determinare ed interpretare le sensazioni provenienti dall'organismo durante un esercizio fisico (Noble \& Robertson 1996). Ciò viene stimato secondo uno specifico metodo di quantificazione, definito scala delle RPE di Borg (Borg 1982).

In letteratura scientifica vengono proposte tre diversi tipi di scale.

1. Borg 6-20 RPE scale ${ }^{\circledR}$ (Borg 1970). È stata progettata per fornire dei dati di facile comprensione e che hanno una relazione lineare con alcuni parametri fisiologici o di lavoro espresso che durante uno sforzo crescono in modo lineare ovvero (e.g.) la frequenza cardiaca e la potenza espressa (Groslambert \& Mahon 2006).

2. Category Ratio Scale $\left(C R-10^{\circledR}\right)$ (Borg 1982). Risulterebbe essere più appropriata per valutare sensazioni che possono derivare da alcune variabili fisiologiche che durante uno sforzo crescono in maniera esponenziale, come il lattato ematico o la ventilazione polmonare (Groslambert et al., 2006). 
3. Category Ratio Scale (CR-100 ${ }^{\circledR}$, o centi Max) (Borg \& Kaijser 2006). Un vantaggio della CR100 ${ }^{\circledR}$ ed anche della CR10 ${ }^{\circledR}$ è che le espressioni verbali consentono di utilizzare questi strumenti sia per la stima sia per la produzione, cioè sia per dare un valore numerico alla propria percezione, sia per dire alle persone a che livello di percezione devono lavorare. La CR100 ${ }^{\circledR}$ mantiene caratteristiche simili alla CR10 ${ }^{\circledR}$ ed è potenzialmente più sensibile.

Sempre Borg sostenne che l'RPE è il ‘... singolo miglior indicatore di uno sforzo fisico ...' ed '... integra varie informazioni tra cui molti segnali ricavati dal lavoro dei muscoli periferici e dalle articolazioni, dal sistema cardiovascolare centrale e polmonare e dal sistema nervoso centrale ...' (Borg 1982). Dunque, la sensazione di sforzo determinata durante un esercizio altro non è che la manifestazione verbale e cosciente dell'integrazione di questi fattori sia di tipo psicologico che di tipo fisiologico. Il collegamento biologico tra le sensazioni soggettive relative ad uno sforzo e le modificazioni fisiologiche che avvengono durante un esercizio sono di estrema importanza, perché forniscono un meccanismo di regolazione della performance stessa, ed il tutto potrebbe essere fatto mediante l'utilizzo dell'RPE (Tucker 2009). 


\section{Determinazione del Carico Allenante}

Quanto detto nelle sezioni precedenti permetterebbe tuttavia solo di stimare l'intensità di un determinato sforzo. Ben diverso è il voler cercare di valutare il carico interno a seguito della somministrazione di un carico esterno. In letteratura sono stati proposti diversi metodi per la quantificazione del carico interno partendo (e.g.) dalla frequenza cardiaca e dalla percezione dello sforzo. Essi sono:

1. Banister's Training Impulse (TRIMP) (Banister 1991).

2. Edwards' Heart Rate-based method (Edwards 1993).

3. Lucia's TRIMP (Lucia, Hoyos, Santalla, Earnest \& Chicharro 2003).

4. Tempo passato in zone d'intensità delimitate dalla frequenza cardiaca di soglia lattacida o ventilatoria (Gilman 1996; Gilman \& Wells 1993).

5. Session-RPE (Foster 1998; Foster, Hector, Welsh, Schrager, Green \& Snyder 1995).

\section{Banister's Training Impulse (TRIMP)}

Banister et al. proposero un metodo per quantificare una sessione di allenamento come un'unita (i.e., dose) di sforzo fisico (Banister 1991). Suggerirono che la risposta soggettiva della frequenza cardiaca ad un determinato esercizio (nel suo insieme chiamato impulso allenante, in inglese training impulse - TRIMP), potesse essere una misura plausibile dello sforzo fisico, in quanto essa si basava sull'ampiezza con cui un esercizio eleva la frequenza cardiaca da una condizione di riposo fino ai massimi livelli (Banister 1991; Morton, Fitz-Clarke \& Banister 1990). Questo metodo si avvale di un calcolo che prevede l'utilizzo della durata dell'allenamento, della massima frequenza cardiaca, della frequenza cardiaca a riposo e della frequenza cardiaca media registrata durante lo sforzo (Equazione 3): 


\section{TRIMP $=$ durata dell allenamento $(\min ) \cdot \Delta$ HRratio $\cdot \mathrm{Y}$}

dove

$\Delta$ HRratio $=\frac{\mathrm{HR}_{\mathrm{ex}}-\mathrm{HR}_{\text {rest }}}{\mathrm{HR}_{\max }-\mathrm{HR}_{\text {rest }}}$

e dove $\mathrm{Y}=0.64 \mathrm{e}^{1.92 \mathrm{x}}$ per $\mathrm{i}$ maschi, $\mathrm{Y}=0.86 \mathrm{e}^{1.67 \mathrm{x}}$ per le femmine, $\mathrm{e}=2.712$ e $\mathrm{X}=\Delta$ HRratio (Equazione 3) (Banister 1991). Tuttavia, questo metodo non sembrerebbe essere privo di limitazioni in quanto limitato dalla necessità dell'utilizzo di cardiofrequenzimetri. Inoltre, vi sarebbe la necessità di raggiungere misure di frequenze cardiache in condizioni di steady-state, il che limiterebbe l'accuratezza con la quale un esercizio a natura intervallata possa essere monitorato e quantificato (Borresen et al., 2009).

\section{Edwards' Heart Rate-based method}

Questo metodo è una modifica del calcolo del TRIMP che faciliterebbe la quantificazione dell'allenamento intervallato (Edwards 1993). Esso determina il carico interno che si ottiene moltiplicando la durata (in minuti) dell'allenamento passato in ciascuna classe di frequenza cardiaca (come riportato a breve) per un coefficiente relativo ad ogni singola zona e successivamente sommare i risultati ottenuti (Edwards 1993):

- $\quad 50-60 \%$ della $\mathrm{HR}_{\max }=1$

- $\quad 60-70 \%$ della $\mathrm{HR}_{\max }=2$

- $70-80 \%$ della $\mathrm{HR}_{\max }=3$

- $\quad 80-90 \%$ della $\mathrm{HR}_{\max }=4$ 
- $\quad 90-100 \%$ della $\mathrm{HR}_{\max }=5$

Tuttavia, questo metodo sembrerebbe non essere privo di limitazioni, in quanto sembrerebbe sovrastimare il reale carico allenante se paragonato ad una stima più soggettiva della risposta ad un carico esterno (i.e., RPE) (Borresen \& Lambert 2008). Sempre gli stessi autori hanno evidenziato come non ci siano evidenze scientifiche sulla validazione di questo metodo. Di conseguenza, questo metodo sembrerebbe essere derivato solo a livello teorico e non attraverso sperimentazione (Borresen et al., 2009).

\section{Lucia's TRIMP}

Questo metodo risulta simile a quello proposto da Edwards; il carico interno viene determinato moltiplicando la durata (in minuti) dell'allenamento in 3 diverse classi di frequenza cardiaca per un coefficiente relativo ad ogni singola zona e successivamente i risultati ottenuti vengono sommati (Lucia et al., 2003). La differenza tra i due metodi risiede nella natura di queste zone di frequenza cardiaca. Se con il metodo di Edwards queste zone sono arbitrarie, col metodo di Lucia esse vengono definite in base a delle caratteristiche soggettive, in relazione alla soglia ventilatoria (SV) ed al punto di compensazione respiratorio (PCR) (Lucia et al., 2003):

- $\quad<\mathrm{SV}=1$

- $\mathrm{SV}-\mathrm{PCR}=2$

- $\quad$ PCR $=3$

Questo metodo di quantificazione condivide le stesse limitazioni dei precedenti, in quanto l'andamento lineare del carico non rispecchierebbe in maniera accurata l'aumento del carico di lavoro stesso che si assisterebbe per intensità al di sopra della soglia anaerobica (Stagno, Thatcher \& van Someren 2007). La soglia anaerobica varia a livello inter-individuale seppur in presenza di 
medesime potenze espresse; di conseguenza lo stress metabolico prodotto dagli individui può essere differente anche allenandosi alle stesse percentuali di frequenza cardiaca (Stagno et al., 2007).

\section{Tempo passato in zone d'intensità delimitate dalla frequenza cardiaca di soglia lattacida o ventilatoria}

Come per gli altri metodi sopra descritti, anche questo determina il carico interno moltiplicando la durata (in minuti) dell'allenamento in diverse classi di frequenza cardiaca (Gilman 1996; Gilman et al., 1993). Queste classi vengono determinate in relazione a due markers metabolici che vengono valutati precedentemente in laboratorio:

1. La Soglia Ventilatoria.

2. L'OBLA alle $4 \mathrm{mmol} \cdot \mathrm{L}^{-1}$.

Le frequenze cardiache successivamente determinate durate lo sforzo vengono usate come riferimento per quantificare l'ammontare dell'allenamento svolto a:

1. Alta Intensità (> HR-OBLA).

2. Intensità Moderata (< HR-OBLA, $>$ HR-VT).

3. Bassa Intensità $(<\mathrm{HR}-\mathrm{VT})$.

Tuttavia, come per il Lucia's TRIMP sopra descritto, questo metodo presenta le medesime limitazioni (Stagno et al., 2007).

\section{Session-RPE}

Nel tentativo di semplificare ulteriormente la quantificazione del carico allenante, Foster et al. hanno introdotto l'utilizzo della Session-RPE (Foster 1998; Foster, Daines, Hector, Snyder \& Welsh 1996; Foster, Florhang, Franklin, Gottschall, Hrovatin, Parker et al., 2001; Foster et al., 1995). Questa misura è un 
valore della difficoltà complessiva dell'esercizio svolto ottenuto 30 minuti dopo aver completato l'esercizio stesso (i.e., per eliminare la possibile influenza dell'ultimo sforzo compiuto sulla percezione dello sforzo complessivo). Ciò è reso possibile dall'equazione 4:

Session-RPE = RPE · durata dello sforzo $(\mathrm{min})$ (Equazione 4) (Foster 1998; Foster et al., 1996;

Foster et al., 2001; Foster et al., 1995)

Questo prodotto rappresenta, in un singolo numero, il magnitudo del carico interno allenante in un'unità arbitraria (AU) (Impellizzeri et al., 2004).

L'uso della Session-RPE per quantificare il carico interno risulterebbe essere potenzialmente indipendente sia dal modo che dall'intensità dello sforzo stesso e potrebbe essere utilizzata per molteplici esercizi (e.g.) ad alta intensità, non steady-state (i.e., esercizi di forza), esercizi intervallati, pliometrici etc. (Foster et al., 2001). Tuttavia, anche questo metodo non si esima dall'avere alcune limitazioni in quanto la RPE sembrerebbe essere influenzata più dal carico di forza che dal volume, di conseguenza (e.g.) eseguire più ripetizioni con un carico leggero sarebbe percepito come meno intenso rispetto che all'opposto (Day, McGuigan, Brice \& Foster 2004; Sweet, Foster, McGuigan \& Brice 2004). Inoltre, l'ordine in cui gli esercizi vengono eseguiti, le fibre muscolari utilizzate, il livello di esperienza dell'atleta ed il momento in cui viene somministrata la RPE possono, a loro volta, influenzare la RPE stessa (Day et al., 2004; Sweet et al., 2004). 


\section{PACING STRATEGY}


Nel tentativo di aumentare la conoscenza della performance atletica (qualsiasi essa sia), la letteratura scientifica, soprattutto nell'ultimo decennio, ha fatto passi enormi nell'analisi di come il lavoro (o l'energia consumata) viene distribuita durante un compito motorio (Foster, deKoning, Hettinga, Lampen, Dodge, Bobbert et al., 2004; Foster, Hoyos, Earnest \& Lucia 2005; Marino 2004; Tucker, Marle, Lambert \& Noakes 2006; van Ingen Schenau, de Koning \& de Groot 1992). Questa distribuzione dello sforzo (o modello di spesa energetica) è stata definita con il termine di pacing strategy (Atkinson, Davison, Jeukendrup \& Passfield 2003; de Koning, Bobbert \& Foster 1999; Foster et al., 2004). Attraverso lo studio di questa tematica, si è potuto riconoscere come la pacing strategy di un atleta (i.e., come un atleta distribuisce il suo lavoro o l'energia durante uno sforzo), può avere un impatto significativo nella performance (Abbiss \& Laursen 2008). È importante sottolineare che il termine pacing strategy riflette in maniera più accurata il tempo (o la velocità) con cui si è compiuta la performance e non il lavoro meccanismo istantaneo o la potenza espressa. Tuttavia, il meccanismo di regolazione è estremamente influenzato dall'abilità di resistere alla fatica, rendendo di conseguenza la potenza meccanica espressa di estrema importanza anche nello studio della pacing strategy (de Koning et al., 1999). Conseguentemente, attraverso lo studio della relazione potenza/velocità e l'osservazione delle performance di atleti di alto livello, sia gli allenatori che i ricercatori sono stati in grado di migliorare le conoscenze specifiche della distribuzione dello sforzo (i.e., pacing strategy ottimale) durante competizioni di varia natura (Arsac \& Locatelli 2002; van Ingen Schenau et al., 1992; Wilberg \& Pratt 1988). Queste possono essere classificate come (Abbiss et al., 2008):

- All-Out Pacing Strategy.

- Negative Pacing Strategy.

- Positive Pacing Strategy. 
- Even Pacing Strategy.

- Parabolic-Shaped Pacing Strategy.

- Variable Pacing Strategy.

\section{All-Out Pacing Strategy}

Durante determinate eventi, il costo associate all'accelerazione di un corpo può significativamente influenzare la pacing strategy richiesta per avere una performance ottimale (van Ingen Schenau et al., 1992). Ad esempio, durante una gara di 100 metri, gli atleti top-level spendono circa il 50-60\% della gara nella fase di accelerazione. Di conseguenza, il 20-25\% del lavoro totale richiesto da questa competizione può essere dovuto semplicemente ad alterare l'energia cinetica del corpo dalla condizione di riposo. Inoltre, dato l'aumento dell'energia cinetica come risultato dell'aumento del momentum, l'energia richiesta per mantenere costante lo sforzo risulta essere inferiore rispetto a quella richiesta per accelerare, specialmente quando l'inerzia è elevata (i.e., grande massa e velocità) (Abbiss et al., 2008). Poiché la spesa energetica richiesta per accelerare è inevitabile, si ritiene che questa energia sia meglio distribuita all'inizio di un evento di breve durata, giacché qualsiasi azione sub-massimale porterebbe ad avere una performance peggiore (de Koning et al., 1999; van Ingen Schenau et al., 1992). Dato che questa accelerazione iniziale è proporzionale alla durata dell'evento, è possibile che la performance ottimale possa essere ottenuta quando gli atleti partono e continuano l'evento stesso con un modello all-out (Bishop, Bonetti \& Dawson 2002; van Ingen Schenau et al., 1992).

\section{Negative Pacing Strategy}

Un evento si considera eseguito con una negative pacing strategy quando c'è un incremento della velocità osservata durante l'evento stesso (Abbiss et al., 2008). L'adozione di questo modello di pacing strategy potrebbe portare ad un miglioramento delle performance di lunga durata mediante (e.g.) (i) la riduzione della deplezione di carboidrati (Abbiss \& Laursen 2005), (ii) un consumo di 
ossigeno inferiore (Sandals, Wood, Draper \& James 2006) e/o (iii) la limitazione dell'accumulo precoce di metaboliti della fatica (Mattern, Kenefick, Kertzer \& Quinn 2001; Robinson, Robinson, Mountjoy \& Bullard 1958).

\section{Positive Pacing Strategy}

$\mathrm{Al}$ contrario della negative pacing strategy, si ha una positive pacing strategy quando la velocità con cui un atleta compie una determinata performance diminuisce progressivamente lungo la durata dell'evento stesso (Abbiss et al., 2008). L'adozione di questo tipo di pacing porta ad avere un aumento del consumo di ossigeno (Sandals et al., 2006; Thompson, MacLaren, Lees \& Atkinson 2003), un maggior accumulo di metaboliti della fatica e, di conseguenza, una maggiore percezione dello sforzo nelle fasi iniziali della competizione (Thompson et al., 2003; Thompson, MacLaren, Lees \& Atkinson 2004).

\section{Even Pacing Strategy}

Come detto poc'anzi, la scelta della strategia con cui un atleta inizia la sua performance può drasticamente influenzare la performance complessiva, specialmente durante eventi di breve durata. Durante eventi di più lunga durata, invece, la scelta con cui iniziare la gara sembrerebbe avere un minore impatto sulla performance totale (Foster et al., 2004; Foster, Schrager, Snyder \& Thompson 1994). Questo grazie al fatto che il tempo speso soprattutto nella fase di accelerazione risulterebbe ridotto. Di conseguenza, è stato suggerito dalla letteratura scientifica che in condizioni esterne controllate (sia ambientali che geografiche), una distribuzione dello sforzo costante sarebbe ottimale per eventi prolungati nel tempo (i.e., > 2 minuti) (de Koning et al., 1999; Thompson et al., 2003, 2004). Minimizzare la variazione della distribuzione dello sforzo può essere determinante soprattutto in quelle discipline in cui la resistenza di un fluido (e.g., aria, acqua) gioca un ruolo importante (i.e., ciclismo, nuoto). 


\section{Parabolic-Shaped Pacing Strategy}

Il recente sviluppo di strumenti di misurazione sia del tempo che della potenza più accurati e ripetibili ha permesso agli scienziati di esaminare in maniera ancora più specifica il profilo di una performance in condizioni reali (i.e., real settings). Ci si è accorti pertanto che gli atleti possono ridurre progressivamente la loro velocità durante un evento, per poi aumentarla di nuovo nell'ultimo tratto dell'evento stesso. Questa tattica di gara porta ad avere delle pacing strategy con caratteristiche a U, J o J rovesciata (Garland 2005; Kennedy \& Bell 2003).

\section{Variable Pacing Strategy}

La ricerca scientifica all'interno della pacing strategy può essere complicata ed influenzata da numerosi fattori esterni come:

- La durata della competizione (Foster et al., 2004).

- La planimetria (Swain 1997).

- Le condizioni Ambientali (e.g.):

○ Vento (de Koning et al., 1999; Padilla, Mujika, Angulo \& Goiriena 2000).

○ Temperatura (Tucker et al., 2006; Tucker, Rauch, Harley \& Noakes 2004).

Di conseguenza, la maggior parte delle ricerche riguardanti la pacing strategy sono state condotte in condizioni ambientali controllate o simulate (Abbiss et al., 2008). Tuttavia, è inusuale per un atleta avere delle condizioni esterne costanti per tutta la durata della propria competizione (Atkinson \& Brunskill 2000). Partendo da questa varietà di condizioni esterne associate con delle performance outdoor, è stato suggerito che delle pacing strategy variabili siano la soluzione tattica più idonea (Atkinson et al., 2000; Liedl, Swain \& Branch 1999). Questo tipo di pacing è un termine che viene usato dalla letteratura scientifica per indicare delle fluttuazioni nell'intensità dell'esercizio o nel 
lavoro prodotto (i.e., potenza espressa) osservate durante una performance (Atkinson et al., 2000; Atkinson, Peacock \& Law 2007; Liedl et al., 1999). Le implicazioni fisiologiche di questo tipo di pacing strategy possono essere estremamente interessanti per la comunità scientifica, poiché (e.g.) un aumento dell'intensità dell'esercizio può significativamente aumentare la richiesta fisiologica di un compito motorio (Sandals et al., 2006; Swain 1997; Thompson, Haljand \& MacLaren 2000; Thompson et al., 2003). 


\section{RESOCONTO DELL'ATTIVITÀ DI DOTTORATO}


Nella marcia, nel corso della sua storia ultra-centenaria, lo stress fisiologico, così come la spesa energetica (i.e., determinants fisiologici) sono stati ampliamente documentati (Brisswalter, Fougeron \& Legros 1998; Farley \& Hamley 1978; Hagberg \& Coyle 1983; Reilly, Hopkins \& Howlett 1979; Yoshida, Udo, Iwai, Muraoka, Tamaki, Yamaguchi et al., 1989). Tuttavia, al fine di comprendere meglio il profilo dello sforzo e la relativa intensità che gli atleti compiono durante le competizioni di marcia (i.e., physiological and regulatory demands) su diverse distanze ufficialmente riconosciute dalla IAAF (i.e., 3 e 5 chilometri indoor; 10, 20 e 50 chilometri outdoor) durante i tre anni del corso di Dottorato di Ricerca si è cercato di incrementare questa conoscenza al fine di migliorare le conoscenze specifiche per poter sviluppare delle strategie di allenamento il più specifiche possibili. 


\section{Physical Activity Intensity, Heart Rate, Blood Lactate Concentration and RPE During 5-Km Men's and 3-Km Women's Indoor Race Walk}

Gianluca Vernillo; Luca Agnello; Andrew Drake; PierLuigi Fiorella; Antonio La Torre

\section{PosterPresentation@14 annual Congress of the EUROPEAN COLLEGE OF SPORT SCIENCE, Oslo/Norway, June 24-27, 2009, pg. 180.}

\section{Introduction}

Physical training is the systematic repetition of physical exercises and it can be described in terms of its outcome (anatomical, physiological, biochemical, and functional adaptations) or its process; that is, the Training Load (TL) (the product of volume and intensity of training). Nowadays, there have been several attempts to quantify TL, such Heart Rate (HR), Blood Lactate Concentration ([La]) and Rate of Perceived Exertion (RPE)[1, 2]. Race Walking (RW) is the technical and athletic expression of fast walking and it can be considered an endurance performance. Despite its centuries-old history few studies have investigated the physiological profile of the race walkers and, moreover, no study has analyzed both the physical activity intensity and the physiological stresses during a race. Thus, this study classified the intensity of the 5-km men's (5M) and 3-km women's (3F) Indoor RW (IRW) and examined both HR, [La] and RPE responses in the two races and the possible relationship between the above mentioned variables in order to verify the Internal Load (IL) of the athletes.

\section{Methods}

11 race walkers, 5 male (mean age: $30,2 \pm 3,4$ yrs; height: $174,6 \pm 3,5 \mathrm{~cm}$; weight: $63,2 \pm 5,9 \mathrm{~kg}$ ) and 6 female (mean age: $22,3 \pm 3,6$ yrs; height: $167,2 \pm 3,3 \mathrm{~cm}$; weight: $53,2 \pm 2,2 \mathrm{~kg}$ ) were enrolled in this 
study. During the Italian Athletic Indoor Championship held in Turin in February 2009, HR was recorded to establish the HRmean; after the performances [La] and RPE (Borg 6-20 scale) were registered. HRmean was set as a percentage of the mean predicted maximal heart rate to quantify, with the RPE, the possible physical effort of the disciplines. Therefore, both for $5 \mathrm{M}$ and $3 \mathrm{~F}$, HRmean, [La] and RPE were standardized with each data point transformed to a $\mathrm{z}$ score $[\mathrm{z}=(\mathrm{raw}$ score-mean)/SD] using the variable specific grand mean and SD. Means for standardized values were then compared using repeated-measures ANOVA. Results were considered significant at $\mathrm{p} \leq 0.05$.

\section{Discussion/Conclusion}

HRmean and the RPE show that both the $5 \mathrm{M}$ and $3 \mathrm{~F}$ present a very hard intensity profile[2], suggesting that the physiological model of the two disciplines might be aerobic-power-oriented. No statistical differences ( $p>0.05)$ were found between the HRmean, [La] and RPE in the two groups; that means in the IRW there might not be a more appropriate tool to evaluate the IL of the athletes; thus the three means seem to be able to measure, indirectly, the IL during an IRW race and the choice might be attributed to the specific knowledge and/or techniques available to the coach. However, considering the low number of the athletes involved, further studies should be conducted to outline the most suitable tool to assess the IL in the IRW.

\section{References}

[1] Achten \& Jeukendrup. Sports Med 2003; 33(7): 517-538

[2] ACSM. Med Sci Sports Exerc 1998; 30(6): 975-991 
The Training for the 20-Km RW. What Is Changed and What Might Change?

Antonio La Torre, Luca Agnello, PierLuigi Fiorella, Gianluca Vernillo

\section{Oral Presentation@2009BASES Annual Conference.J Sports Sci (2009), 27: 4, S18}

The race walk (RW) can be described as the technical and athletic expression of fast walking. Despite its centuries-old history few studies have investigated the physiological profile of the RW athlete (Hagberg \& Coyle, 1983: Medicine and Science in Sports and Exercise, 15, 287-289; Yoshida et al., 1989: British Journal of Sports Medicine, 23, 250-254); therefore, there is insufficient direct scientific evidence to formulate training recommendations useful both for the RW athletes and coaches. This is particularly evident when considering the many methodological factors associated with the RW that matches the common physiological determinants of long-distance performance: (i) maximal oxygen uptake ( $\left.\dot{\mathrm{V}}_{2 \text { max }}\right)$ (Noakes et al., 1990: Journal of Sports Science, 8, 35-45; Basset \& Howley, 2000: Medicine and Science in Sports and Exercise, 32, 70-84); (ii) lactate threshold (LT) (Noakes et al., 1990: Journal of Sports Science, 8, 35- 45; Tanaka \& Matsuura, 1990: Journal of Applied Physiology, 57, 640-643); (iii) running economy (RE) (Conley \& Krahenbuhl, 1980: Medicine and Science in Sports and Exercise, 12, 357-360; Noakes et al., 1990: Journal of Sports Science, 8, 35-45; Basset \& Howley, 2000:Medicine and Science in Sports and Exercise, 32, 70- 84) and (iv) percent of maximum oxygen uptake $\left(\% \dot{\mathrm{V}} \mathrm{O}_{2 \max }\right.$ ) (Basset \& Howley, 2000: Medicine and Science in Sports and Exercise, 32, 70-84), with the technical and regulations aspects. This doesn't mean that the scientific literature cannot provide valid support to enhance the performance in the RW, but just that there is a lack of specific knowledge; consequently, the ability of the coaches should be to shift the main findings of the research into the RW training. Thus, the purpose of this report has been to analyse the most topical results concerning the physiological adaptations of 
different training methods for the endurance disciplines that, in our opinion, might be useful for the RW training as well, referring to practical experiences through the years.

\section{Pacing Strategy During 50-Km Race Walking Events}

Gianluca Vernillo; Franco Impellizzeri; Antonio La Torre

Oral Presentation@15 annual Congress of the EUROPEAN COLLEGE OF SPORT SCIENCE, Antalya/Turkey, June 23-26, 2010, pg. 45.

\section{Introduction}

The gait employed during race walk (RW) is a function of the rules which govern this sport (i.e. not fly-time and bent knee). During a competition the athletes must adhere to these or face disqualification after received 3 technical warnings (TW). Thus, pacing strategy (PS) during RW events may be also affect by TW, influencing the overall distribution of the effort. Accordingly, the aim of this study was to analyze the effects of TW on the performance during a $50-\mathrm{km}$ RW race.

\section{Methods}

Total times and 5-km split times were obtained from the official race results of the $50-\mathrm{km}$ RW during the XII World Championship 2009. The 31 athletes which finished the race were divided in 3 groups according to TW received (0-, 1-, and 2-TW, respectively). Alterations in PS and its interactions were determined by two-way ANOVA (group x distance). The velocity during each 5$\mathrm{km}$ section was compared with average racing velocity using a one-way ANOVA. When a significant F-value was found, a Bonferroni post hoc test was applied. Data are presented as mean \pm SD and the level set for significance was $\mathrm{p}<0.05$.

\section{Results}


Walking velocity was not significantly different between the groups both at each $5-\mathrm{km}$ and over the entire race distance $(\mathrm{p}>0.05)$. The velocity vs. distance curve showed a positive strategy[1] with the last two $5-\mathrm{km}$ sectors significantly different compared to the previous $(\mathrm{p}<0.05)$ for the all groups. In fact, until $40-\mathrm{km}$ athletes walked at an average velocity of $13.0 \pm 0.4,13.1 \pm 0.1$, and $12.9 \pm 0.1 \mathrm{~km} \cdot \mathrm{h}^{-1}$, respectively; while, the average walking velocity during the last two sectors decreased $\left(11.9 \pm 0.2,12.2 \pm 0.2\right.$, and $11.8 \pm 0.02 \mathrm{~km} \cdot \mathrm{h}^{-1}$, respectively).

\section{Discussion/Conclusion}

This analysis suggests that PS is unaffected by TW. An explanation for this may be that PS is set before commencement of the event (i.e. in an anticipatory manner) by the athletes based on expectations of exercise duration[2] and this setting is not influenced by the risk of disqualification. The sudden decline of the athletes' walking velocity throughout the last two 5 -km sectors suggests a failure in the ability to race at an optimal intensity which implied that all available energy sources were used before the end of the race, in such a way that a drastically decrease in overall speed occurs[3]. Further researches are required to understand the influence of TW on the physiological strain during RW races on a moment-to-moment basis.

\section{References}

[1] Abbiss \& Laursen (2008). Sports Medicine 38(3):239-52

[2] Albertus et al. (2005). Med Sci Sports Exerc 37(3):461-68

[3] Tucker \& $\quad$ Noakes TD (2009). $\quad$ Br $\quad J \quad$ Sports $\quad$ Med 2009 43(6):e1 
Technical Warnings-Related Effects on the Performance During a 50-Km Race Walk Competition

Gianluca Vernillo; Luca Agnello; Andrew Drake; Maria Francesca Piacentini; Antonio La Torre

\section{PosterPresentation@15 annual Congress of the EUROPEAN COLLEGE OF SPORT SCIENCE, Antalya/Turkey, June 23-26, 2010, pg. 175.}

\section{Introduction}

The ability of an athlete to race at an optimal intensity to avoid an excessive fatigue is a crucial factor to optimize the performance. This distribution of work is commonly defined as pacing strategy[1]. To date no study concerning pacing strategy during $50-\mathrm{km}$ race walk events has been conducted. Thus, the aim of this study was to investigate the pacing strategy adopted by athletes during international events (IE) as well as the challenge events (CE).

\section{Methods}

510 total times and 5-km split times were obtained from official race results of 16 races (12 IE and 4 CE). Assumptions of normality were verified using the Kolmogorov-Smirnov test. A one-way ANOVA was used to assess differences in 5-km split time of the two groups. When a significant Fvalue was found, a Bonferroni post hoc test was applied. Unpaired student's t-test was applied to determine differences in the total time and split time between IE and CE. Data are presented as mean \pm SD and the level set for significance was $\mathrm{p}<0.05$. 


\section{Results}

The mean finishing times were $3 \mathrm{~h} 46 \mathrm{~min} 08 \mathrm{~s} \pm 02 \mathrm{~min} 43 \mathrm{~s}$ and $4 \mathrm{~h} 04 \mathrm{~min} 36 \mathrm{~s} \pm 05 \mathrm{~min} 13 \mathrm{~s}$ for IE and CE, respectively. There were significant differences in 5-km split time in the two groups; in fact after an initial acceleration significant decays in the latter portion of the events were observed. Total times and split times between IE and CE were significantly different.

\section{Discussion/Conclusion}

IE and CE were characterized by a different pacing profile, adopting reverse U-shaped and positive pacing, respectively[2]. Thus, athletes tend to adopt both a positive and negative pacing strategy in contrast with literature that suggests adoption of a more even or varied pacing strategy for prolonged events[2]. This regulation has to be dictated by the ability of an athlete to resist fatigue, even if the precise mechanisms responsible are currently unclear[2]. This is particularly evident in the mean pacing: total time and split-times in CE were significantly higher than IE, probably explained by a (i) lower level of the athletes involved in CE events, and (ii) a limited number of $\mathrm{CE}$ events. Further research is required in order to find out (i) contributions of central and peripheral fatigue during these exercise tasks, and (ii) the influence of external factors (i.e. technical warnings) to the regulation of pace.

\section{References}

[1] Foster et al. (2004). Int J Sports Med 25(3):198-204

[2] Abbiss \& Laursen (2008). Sports Medicine 38(3):239-52 


\section{Quantifying the Training Load During a 10000-m Race Walk}

Gianluca Vernillo; Luca Agnello; Maria Francesca Piacentini; Antonio La Torre

\section{Poster \& Oral Presentation@2 $2^{\text {nd }}$ National Congress of the SOCIETÁ ITALIANA delle SCIENZE MOTORIE e SPORTIVE, Turin/Italy, September 29 October 2, 2010. J Sports} Med Phys Fitness (2010), 50: 3, S22

Training load (TL) is the product of intensity, duration and frequency of a training session. Among the various methods proposed the one suggested by Edwards is a valid manner to assess the heart rate (HR)-based TL. It is the product of the accumulated training duration (minutes) of 5 HR zones by a coefficient relative to each zone $(50-60 \%$ of $H R \max =1,60-70 \%$ of $\mathrm{HRmax}=2,70$ $80 \%$ of HRmax $=3,80-90 \%$ of HRmax $=4,90-100 \%$ of HRmax $=5$ ) and then summating the results. Consequently, the aim of this study was to assess the HR-based TL during a 10000-m race walk competition. 14 male race walkers (mean \pm SD: age $25.1 \pm 5.1 \mathrm{yr}$; height: $177.1 \pm 6.1 \mathrm{~cm}$; weight: $64.2 \pm 4.4 \mathrm{~kg}$ ) were enrolled in this study. During a $10-\mathrm{km}$ race performed on a certified 400-m outdoor-tartan-track HR was recorded continuously. HRmax was taken to be the highest HR achieved during the race and this value was used in the aforementioned formula. . The mean final time, HRmax and TL were $44 \mathrm{~min} 16 \mathrm{~s} 06$ tenths $\pm 2 \mathrm{~min} 27 \mathrm{~s} 21$ tenths, $189.4 \pm 6.8$ beats/min and $220.4 \pm 11.5$ arbitrary unit (AU), respectively. Furthermore, athletes spent $95.38 \%(42 \mathrm{~min} 13 \mathrm{~s})$ at 90-100\% of HRmax, the other work $(4.62 \%$, 02min 03s) was negligible. Although this formula represents in a single number the magnitude of internal TL in AU, to monitor and control the training process it is important to have a valid measure even during competitions, to be able to compare these values with the same found through the training periodisation. Consequently, these data can represent a valid example of internal TL which is sustained by the athletes during this kind 
of race. Moreover, the HR-based TL method proposed above combined with the HR monitoring could be considered a valuable tool for coaches and athletes to monitor internal TL and the physiological processes during every single training session in race walkers. 
Variations of Perceived Exertion in Relation to Heart Rate Responses in an International Level Race Walkers Before a 50-Km Race

Gianluca Vernillo; Franco Impellizzeri; Patrizio Parcesepe Antonio La Torre

\section{PosterPresentation@16 annual Congress of the EUROPEANCOLLEGE OF SPORT SCIENCE, Liverpool/UK, July 6-9, 2011, pg. 450.}

\section{Introduction}

The ability to accurately control and monitor exercise intensity (EI) during training sessions plays a key role in planning the athletic season. This can be achieved by means of rating of perceived exertion (RPE) (Borg, 1998) and heart rate (HR) (Achten and Jeukendrup, 2003). Over the years several RPE- and HR-based methods have been proposed to quantify global EI. However, to date no study has been conducted with regards to race walking (RW). Thus, this case study aimed to investigate the typical RPE and HR seasonal variations of an international level race walker training for a 50-km race and to examine the relationship between RPE and HR to further confirm the use of RPE to assess EI during RW-specific training.

\section{Methods}

Training data were collected during the competitive season 2009 (127 training sessions) consisting of four macrocycles of intense training interspersed by one week of tapering before the Italian 50-km championship. The Borg's 6-20 RPE scale (Borg, 1998) was used to measure the athlete perception of effort referred to the whole training session. HR was recorded using a shortrange telemetry system and expressed relative to the maximum value (\%HRmax) of the athlete determined during an incremental maximal test. The relationships between RPE and \%HRmax were analysed using Pearson's product moment correlation. Statistical significance was set at $\mathrm{P}<0.05$. 


\section{Results}

The athlete completed the event in $4 \mathrm{~h} 14 \mathrm{~min} 02 \mathrm{~s}$ (personal best time, $39 \mathrm{~min} 48 \mathrm{~s}$ slower than the World Record). The mean RPE was 11.1 (1.6), 13.1 (1.3), 13.2 (1.5), 12.4 (1.3) and $11.4(1.0), 13.5$ (0.8), 12.7 (0.4), 12.0 (1.5) for the training and tapering period, respectively. The mean \%HRmax was 83.7 (2.0), 85.3 (4.6), 84.8 (4.1), 85.7 (3.8) and 83.0 (0.7), 85.7 (4.5), 84.8 (1.6), $85.4(2.5) \%$ for the training and tapering period, respectively. The correlation between the mean RPE and \%HRmax showed a very large significant relationship $(r=0.74, \mathrm{P}=0.04)$.

\section{Discussion/Conclusion}

The result found indicates that a very large and significant relation exists between RPE and HR. This finding is in line with previous studies which showed how RPE is correlated with many physiological variables, such as HR (Chen et al., 2002), and provides evidence which confirms that RPE can be considered a simple and valid method for quantifying the global EI during RW-specific training.

\section{References}

Achten J, Jeukendrup AE. (2003). Sports Med, 33, 517-538

Borg G. (1998). Borg's perceived exertion and pain scale. Human Kinetics, Champaign, IL

Chen MJ, Fan X, Moe ST (2002). J Sports Sci, 20, 873-899 
An Observational Study on the Perceptive and Physiological Variables During a 10,000-m Race Walking Competition (Vernillo, Agnello, Drake, Padulo, Piacentini \& Torre)

In this study, we observed the variations on physiological and perceptual variables during a selfpaced 10,000-m race walking (RW) event with the aim to trace a preliminary performance profile of the distance. In 14 male athletes, the heart rate (HR) was monitored continuously throughout the event. The rating of perceived exertion (RPE) was collected using the Borg's 6-20 RPE scale placed at each 1,000 $\mathrm{m}$ of an outdoor tartan track. Pacing data were retrieved from the official race results and presented as percent change compared with the first split time. The athletes spent $95.4 \%$ at $90-$ $100 \%$ of the HRpeak, whereas the other work (4.6\%) was negligible. During the race, a shift toward higher HR values was observed because \% HRpeak increased by $3.6 \%$ in the last vs. the first $1,000-\mathrm{m}$ sector $(\mathrm{p}=0.002$, effect size $[\mathrm{ES}]=1.5560 .68$,large $)$. The mean RPE reported by the athletes in the last1,000 $\mathrm{m}$ was significantly higher than in the first 5 sectors $(\mathrm{p}, 0.02, \mathrm{ES}=1.93-2.96$, large to very large). The mean percent change increased between the first 6 sectors and the last $1,000-\mathrm{m}$ sector ( $\mathrm{p}$, $0.01, \mathrm{ES}=1.02-2.1$, moderate to very large). The analysis of walking velocity at each $1,000-\mathrm{m}$ sector suggested the adoption of a negative pacing. In conclusion, the RPE may be a valid marker of exercise intensity even in real settings. Match physiological and perceptual data with work rate are required to understand race-related regulatory processes. Pacing should be considered as a conscious behavior decided by the athletes based on the internal feedback during the race. 


\section{Cardiovascular Responses During an Indoor Race Walking Competition}

Gianluca Vernillo, Luca Agnello, Andrew Drake, Pierluigi Fiorella, Maria F. Piacentini, Antonio La Torre

\section{J Sports Med Phys Fitness. Manuscript Under Revision}

Aim. This study aims to examine the cardiovascular responses during an indoor race walking competition over the distance of 3-km for female and 5-km for male athletes.

Methods. During the Italian indoor RW Championship heart rate was monitored on eleven well trained race walkers (five men and six women) and then refereed as percentages of individuals' theoretical maximum heart rate $(206-0.7 \cdot a g e)$. To provide a measure of relative intensity, five HR zones were assessed. Alterations in \% HRmax both for the five and three $1000-\mathrm{m}$ split distances were determined.

Results. During the 5-km race the athletes spent 79.7\% (15 min 45 s) at HR5 (i.e., $90-100 \%$ of HRmax). Specifically, \% HRmax increased by $10 \%$ in the last compared to the first 1000 -m sector $(\mathrm{P}=0.006$, effect size $=2.47 \pm 0.83$, very large $)$, with the first $1000-\mathrm{m}$ sector lower than the subsequent ones $(\mathrm{P}=0.01$, effect size $=2.17$ to 2.47 , very large). While, for the 3 - $\mathrm{km}$ the athletes spent $86.9 \%$ (11 $\min 35$ s) at HR5 (i.e., $90-100 \%$ of HRmax) with no differences observed in the $\%$ HRmax between the three 1000-m sectors ( $\mathrm{P}>0.01)$.

Conclusion. The dissemination of performance and physical attributes identified within the present study reveal that the exercise intensity of indoor race walking competitions has a highintensity profile and will assist coaches and athletes in formulating appropriate training, competition and recovery. 
Exercise Intensity and PaCing Strategy of A 5KM INDOOR RACE WALK DURING A WORLD RECORD ATTEMPT: A CASE STUdY. 


\section{INTRODUCTION}

Race walk (RW) can be described as the technical and athletic expression of fast walking, adhering to strict technique (International Association of Athletics Federations, IAAF-Rule 230). Consequently, the differences between common walking and RW derive directly from these technical and ruling aspects (e.g., no loss of contact and not bent at the knee from heel strike through to midstance). Traditionally, RW is constituted by indoor ( 5 and $3 \mathrm{~km}$ for men and women, respectively) and outdoor races, which are the Olympic distances $(20 \mathrm{~km}$ for men and women and $50 \mathrm{~km}$ only for men). In particular, indoor RW races (completed on 200-m tracks) are performed during the winter season and can be considered as a bridge between the athlete's general training period (from October to January) and the special training period (from February to mid-May) that come before the major competitions planned throughout the summer (e.g., World Championships or Olympic Games).

Over its centuries-old history, the physiological stress, energy expenditure or physiological determinants, which are important for RW performances, have been extensively investigated (Brisswalter et al., 1998; Farley et al., 1978; Hagberg et al., 1983; Reilly et al., 1979; Yoshida et al., 1989). However, for understanding the physiological stress imposed on the athletes in real setting, it is also necessary to collect and analyze exercise intensity data during actual competitions. This would provide information useful for developing specific training strategies. To the authors' knowledge, there are no studies reporting the exercise intensity during real competitions including outdoor and indoor races.

Among the various methods available, the measure of heart rate (HR) is the most common method used for prescribing and quantifying the exercise intensity (Gilman 1996; Gilman et al., 1993; 
Lamberts \& Lambert 2009; Lamberts, Lemmink, Durandt \& Lambert 2004; Midgley, McNaughton \& Jones 2007). Its validity is mainly related to the well-established linear relation with oxygen uptake (Åstrand et al., 1986). Although some limitations (Achten et al., 2003) exist, the HR can provide important feedback to the coach regarding the training stimulus and the physiological load applied to the athletes (Coutts, Rampinini, Marcora, Castagna \& Impellizzeri 2009; Foster, Green, Snyder \& Thompson 1993). However, for understanding RW performance, the physiological responses provided by HR or other indicators are not sufficient. Indeed, this information must be interpreted in relation to the pacing strategy, that is, the distribution of the effort over the entire event (Abbiss et al., 2008; Foster et al., 2001). Therefore, the combination of HR and pacing strategy may give an additional insight into the underlying physiological and regulatory processes during an indoor RW event.

The aim of this study was to provide data on the exercise intensity and pacing strategy collected during a World Record attempt of a top-level athlete during a 5-km indoor RW race. Therefore, in this case study, we provided unique data about the physiological strain sustainable by an RW athlete during a 5-km indoor race but that were still compatible with a world-class performance. 


\section{METHODS}

\section{Experimental Approach to the Problem}

The purpose of this case study was to measure the physiological strain and work rate in an international class male race walker performing a $5-\mathrm{km}$ indoor RW race with the intent to achieve the World Record. Initially, this study was designed for describing the exercise intensity of a World Record, given that the athlete and coach were confident of his good condition. However, the attempt failed (World Record resisting since 1995). Despite this, the athlete attained the 2009 World leading performance, indicating that he actually was in a good physical condition. Therefore, these data can provide to the coaches and scientists unique data on both the sustainable exercise intensity and pacing strategy, useful to understand if the athlete failed to distribute the effort throughout the race. The pacing strategy was obtained from the data of the official race report supplied by the organizers. During this kind of events, athletes usually prefer to avoid external factors (i.e., HR monitor) that may disturb and negatively influence the performance. In this study, the athlete consented to wear the HR monitor, and therefore, he was previously familiarized with the device. However, other parameters such as rating of perceived exertion could not be collected. The exercise intensity of the race was reported according to a previous method. An incremental laboratory test was used before the competition for characterizing the athlete in terms of aerobic power and to determine the reference HR for establishing the exercise intensity zones (Karvonen et al., 1957). Pacing data were presented as time series and analyzed qualitatively.

\section{Subjects}

The athlete (age: 32 years; height: $1.76 \mathrm{~m}$; body mass: $62 \mathrm{~kg}$; training volume: $130-150 \mathrm{~km} \cdot \mathrm{wk}^{-1}$ ) is an international class male race walker with 20 years of long-term training planning. After he reached an international level of competition, he won the 50-km RW during the VII World 
Championships (Seville 1999); the 20-km RW during the XXVIII Olympic Games (Athens 2004), and he was classified fifth in the 20-km RW during the XXIX Olympic Games (Beijing 2008). His personal best performances among the different RW events are presented in Table 1; particularly, he holds the second all-time performance in the 5-km indoor RW. The athlete performed the 5-km RW at the Italian National Athletic Indoor Championship on February 2009, and the target was to attain the World Record. Specifically, after the getting the fifth place during the Olympic Games in Beijing 2008, he spent 30 days resting and starting the training again in October and focused on the preparation for the XII World Championships 20-km RW (Berlin 2009). During this period, the athlete walked a total distance of 2,100 km with $12 \%(252 \mathrm{~km})$ at high-intensity and $88 \%(1,848 \mathrm{~km})$ at low-intensity (above and under $4 \mathrm{mmol} \cdot \mathrm{L}^{-1}$, respectively). The last high intensity training was performed 5 days before the race, with a tapering strategy consisted of $50 \%$ training reduction in both the volume and intensity.

The athlete was fully informed of the aims and the procedures of the study receiving both verbal and written explanation. Moreover, he gave an informed consent in agreement with the Declaration of Helsinki on human experimentation. This study was approved by the local Ethics Committee.

\section{Procedures}

Laboratory Tests. Two weeks before the competition, maximal oxygen uptake $\left(\mathrm{V}_{2} \max \right)$ was determined on a motorized treadmill (RunRace, Technogym, Gambettola, Italy) at an inclination of $1 \%$. After 3 minutes at $8 \mathrm{~km} \cdot \mathrm{h}^{-1}$, the test began at $9 \mathrm{~km} \cdot \mathrm{h}^{-1}$, and the velocity was increased by $1 \mathrm{~km} \cdot \mathrm{h}$

${ }^{1}$ every 1 minute so that exhaustion was reached in $8-12$ minutes. $\dot{\mathrm{V}} \mathrm{O}_{2}$ max was considered attained according to Howley et al. (Howley et al., 1995). Expired gases were analyzed using a breath-bybreath gas-analysis system (Quark B2, Cosmed, Rome, Italy). Before the test, the calibrations were made according to the manufacturer's recommendations. Moreover, continuous HR was recorded using HR monitor (Polar S610 HR Monitor, Polar Electro Oy, Kempele, Finland). Heart rate 
recordings were averaged for every 5 seconds. The maximal HR value (HRmax) was computed as the maximum HR value obtained during the test.

Data Collection during the Race. On the day of the race, the athlete abstained from ingesting alcohol and caffeine; moreover, no food was ingested 3 hours before the race, while water was supplied ad libitum. Previous to the performance, the athlete carried out a warm-up consisting of 20 minutes of slow RW and 10 minutes of technical drills. The race was performed on a certified $200-\mathrm{m}$ indoor $\operatorname{tartan} \operatorname{track}\left(\sim 20^{\circ} \mathrm{C}, 51 \%\right.$ relative humidity, no wind, $239-\mathrm{m}$ altitude). The HR was measured using a short-range telemetry system. Subsequently, the HRmean and the HRmean/HRmax ratio were assessed, and the reference HRs were calculated from the "Karvonen formula" by multiplying the HR reserve $($ HRR; HRR $=$ HRmax - HRrest $)$ by the factors $0.5,0.7$, and 0.9 and adding these values to the HRrest (19). The following HR ranges were defined: (a) long-term endurance range (HRlte), $<117 \mathrm{~b} \cdot \mathrm{min}^{-1}(<50 \% \mathrm{HRR})$; (b) extensive aerobic range (HRea), 117-147 b·min ${ }^{-1}(50-70 \% \mathrm{HRR})$; (c) intensive aerobic range (HRia), 147-177 b· $\mathrm{min}^{-1}$ (70-90\% HRR); (d) high-intensity range (HRhi), > $177 \mathrm{~b} \cdot \mathrm{min}^{-1}(>90 \%$ HRR) (Gilman 1996; Gilman et al., 1993).

\section{Statistical Analyses}

To describe exercise intensity, HR recordings were expressed relative to the maximum value (\%HRmax) of the athlete observed during the laboratory test. Exercise intensity was also quantified using 4 HR zones previously described and, subsequently, divided into 3 parts (initial, middle, and final). Race distance and walking velocity were expressed in 'actual units' ( $\mathrm{m}$ and $\mathrm{m} \cdot \mathrm{s}^{-1}$, respectively). Pacing strategy was presented as absolute speed every 1,000 $\mathrm{m}$ and as percent change compared to the first split time.

Data are presented as mean \pm SD. Given the nature of the study, only a qualitative analysis has been performed. Pacing and HR data are presented as times series. 


\section{RESULTS}

The athlete's HRrest and HRmax were 42 and $192 \mathrm{~b} \cdot \mathrm{min}^{-1}$, respectively, whereas the $\dot{\mathrm{V}} \mathrm{O}_{2 \max }$ was $70 \mathrm{ml} \cdot \mathrm{kg}^{-1} \cdot \mathrm{min}^{-1}$

The race time was 18 minutes 23 seconds 47 tenths, the 11th performance all-time and the 2009 World leading performance (available at: http://www.iaaf.org). The HRmean recorded and the HRmean/HRmax ratio were $185614.9 \mathrm{~b} \cdot \mathrm{min}^{-1}$ and 0.96 , respectively. The mean distribution of the HR during the performance shows a tight range of intensity in this event. The athlete performed 15, 25, 50 seconds and 16 minutes 55 seconds to intensities corresponding at HRlte, HRlte, HRia, and HRhi, respectively. During the race, a shift toward a higher HR was observed. The first and the second halves of the race (09 minutes 03 seconds 22 tenths and 09 minutes 20 seconds 25 tenths) were performed at HRmean of 179619.5 and $19061.5 \mathrm{~b} \cdot \mathrm{min}^{-1}$, respectively, increasing by $5.7 \%$. The calculated HRmean/HRmax ratios were 0.97 and 0.99 , respectively. A detailed description of the HR response expressed relative to the \%HRmax is illustrated in Figure 1.

As demonstrated in Figure 2, the HR responses expressed relative to the HR ranges showed that the athlete mainly sustained the effort to a high-intensity range (HRhi; $>177 \mathrm{~b} \cdot \mathrm{min}^{-1} ;>90 \% \mathrm{HRR}$ ) both in the 3 parts of the races. In fact, the time spent at HRhi was 04 minutes 33 seconds for the initial part, and 06 minutes 08 seconds for both the middle and final parts. The other ranges (HRlte, HRea, and HRia) were reached only in the initial part.

The pattern of walking velocity is presented in Figure 3. Comparing the pacing strategy every $1,000 \mathrm{~m}$ as percent change of the first split time, the curve showed a classic reverse J-shaped, with a fast start in the first $1,000 \mathrm{~m}\left(4.63 \mathrm{~m} \cdot \mathrm{s}^{-1}, 0 \%\right)$, followed by a gradual decline in walking velocity in 2,000-, 3,000-, and 4,000-m sections (4.59, 20.92\%; 4.50, 22.7\%; and 4,46 $\mathrm{m} \cdot \mathrm{s}^{-1}, 23.57 \%$; respectively), with a final end spurt during the last $1,000 \mathrm{~m}$ of the race $\left(4.48 \mathrm{~m} \cdot \mathrm{s}^{-1}, 23.14 \%\right)$. 


\section{DISCUSSION}

The aim of this case study was to analyze the exercise intensity and pacing strategy during a World Record attempt of a top-level athlete in a 5-km indoor RW race. Even if the attempt failed, these data can provide useful considerations on both the sustainable exercise intensity and pacing strategy.

Primarily, it was observed that the athlete's HR increased in a consistent pattern during the RW competitive event. In fact, almost all the exercise (91.8\%) was performed to an HRhi, with an HRmean of $185614.9 \mathrm{~b} \cdot \mathrm{min}^{-1}$, close to $96 \%$ of the HRmax. The work performed inside the other ranges was negligible because HRlte, HRea, HRia (8.1\%, 90 seconds) were mainly measured during the initial phases of the race (Figure 2), in line with a previous study, which showed that the HR of trained endurance athletes increases in $\sim 90$ seconds from the start of the race (Foster et al., 2004). Although the cardiac output remained nearly constant until the end of the race (as shown in Figure 1), the athlete's walking pace slowed constantly throughout the event. This consideration might suggest that during the race, a cardiovascular drift (CV drift) (Coyle \& Gonzalez-Alonso 2001) occurred. The CV drift is particularly evident if environmental heat stress is high because the hot environment may lead to a drift in several CV responses (Lafrenz, Wingo, Ganio \& Cureton 2008; Wingo, Lafrenz, Ganio, Edwards \& Cureton 2005). In this regard, given the location of the event (i.e., indoor track), the ambient temperature was in stable conditions $\left(\sim 20^{\circ} \mathrm{C}, 51 \%\right.$ relative humidity, no wind), suggesting that during an indoor RW race performed in a neutral environment condition, the CV drifts (e.g., a progressive decline in stroke volume) is probably because of increased HR at a certain walking intensity rather than a progressive increase in dehydration or cutaneous blood flow as body temperature rises (Coyle et al., 2001; Fritzsche, Switzer, Hodgkinson \& Coyle 1999).

Second, the aforementioned considerations support the concept that using only the physiological responses provided by HR for understanding a RW performance is not sufficient to 
describe the stress imposed on the athlete in a real setting, because it needs to reflect the exercise intensity to the distribution of work throughout an exercise task. Indeed, despite the effort being very close to the high-intensity range for the entire race (as previously stated), the pacing strategy adopted by the athlete shows a nonlinear profile, suggesting that the athlete's pacing strategy may be considered as a reverse J-shaped pacing curve (Figure 3), because the athlete adopted both a positive and a negative profile during the race (Abbiss et al., 2008), decreasing and then increasing the speed, respectively. In fact, the first $1,000 \mathrm{~m}$ was performed in the fastest time $(6$ seconds faster than subsequent race sectors), with a reduction in speed observed during the middle 3,000 $\mathrm{m}$, and a new increase (i.e., end spurt) during the final 1,000 $\mathrm{m}$ of the race. Using a mathematical model (AlvarezRamirez 2002) that predicts the maximal aerobic power available during an exercise, we may observe that the maximal requirements for aerobic power would be $97 \%$ and would be achieved at distance between 2,700 and 3,700 $\mathrm{m}$. This distance range could be considered as the most demanding distances from the viewpoint of aerobic metabolism in 5,000-m races.

Third, the irregularity in the distribution of the work over the race was probably related to the athlete's perception of effort, 'a conscious awareness of the central motor commands to the locomotor and respiratory muscles' (Marcora 2009a). Indeed, the athlete's main focus was the attainment of the World Record because both the athlete and coach were confident of his shape; however, after the first split time, the walking velocity started to decrease (Figure 3). This slowing down was mainly related to the athlete's perceived exertion (personal communication) probably higher than the maximum effort set by the athlete to succeed in the exercise task (Marcora 2008). Thus, the athlete overestimated his physical or training status and to avoid a failure in the competition slowed down after the first $1,000 \mathrm{~m}$. This decision seems to prove that pacing strategy, and consequently the exercise performance, is regulated by the conscious brain 'on the basis of the maximum effort a person is willing to exert to satisfy a motive and perceived exertion' (Marcora 2009b). This is also supported by the presence of a final end spurt in the last 1,000 $\mathrm{m}$ (Figure 3). In 
fact, the conscious awareness of exercise task-related end point may allow the athlete to adjust the work rate after the first $1,000 \mathrm{~m}$ so that to economize the power output, preserving the energy stokes available for a possible final spurt.

Finally, before the competition, the athlete performed an incremental laboratory test (mentioned above) to characterize his aerobic power. The result showed that the athlete's $\dot{\mathrm{VO}_{2} \max }$ was $70 \mathrm{ml} \cdot \mathrm{kg}^{-}$ ${ }^{1} \cdot \mathrm{min}^{-1}$, in line with previous research findings among other endurance athletes such as Caucasian(Esteve-Lanao, San Juan, Earnest, Foster \& Lucia 2005) and South African-distance runners (Coetzer, Noakes, Sanders, Lambert, Bosch, Wiggins et al., 1993), and cross-country skiers (Mahood, Kenefick, Kertzer \& Quinn 2001). Furthermore, the athlete’s patterns of training approximates 88\% low-intensity training $\left(<4 \mathrm{mmol} \cdot \mathrm{L}^{-1}\right)$. This training strategy seems to be in line with the concept that endurance athletes spend most of their training time at a low-intensity zone (Esteve-Lanao et al., 2005), even if a $12 \%$ of high intensity training $\left(>4 \mathrm{mmol} \cdot \mathrm{L}^{-1}\right)$ was performed according to other research studies, which stated that physiological and performance development might be related to high-intensity training sessions [for a full review see Ref. (Midgley et al., 2007)]. 


\section{PRACTICAL APPLICATIONS}

The findings of this study suggest using HR monitoring matched with the regulation of the physiologic strain (i.e., pacing strategy) to understand the physiological and regulatory processes during an indoor RW event.

Furthermore, coaches and athletes should be aware that during an indoor 5-km RW, a distribution of the effort with a fast-start strategy (above the athlete's perceived exertion) may lead to a drastic decrease in the power output, compromising the overall performance.

Finally, the presence of a final end spurt suggests that the pacing strategy is a conscious behavior, which is decided by the athlete himself according to his interior feedbacks during the race. 


\section{Table 1}

\begin{tabular}{cc}
\hline Event & Personal Best \\
\hline 5-km Indoor & 18 min 08 s 86 tenths \\
\hline 20-km Outdoor & 1 h 19 min 36 s \\
\hline 50-km Outdoor & 3 h 47 min 54 s \\
\hline
\end{tabular}

Table 1. Personal-best among the standard distances for indoor- and outdoor-races. 


\section{Figure 1}

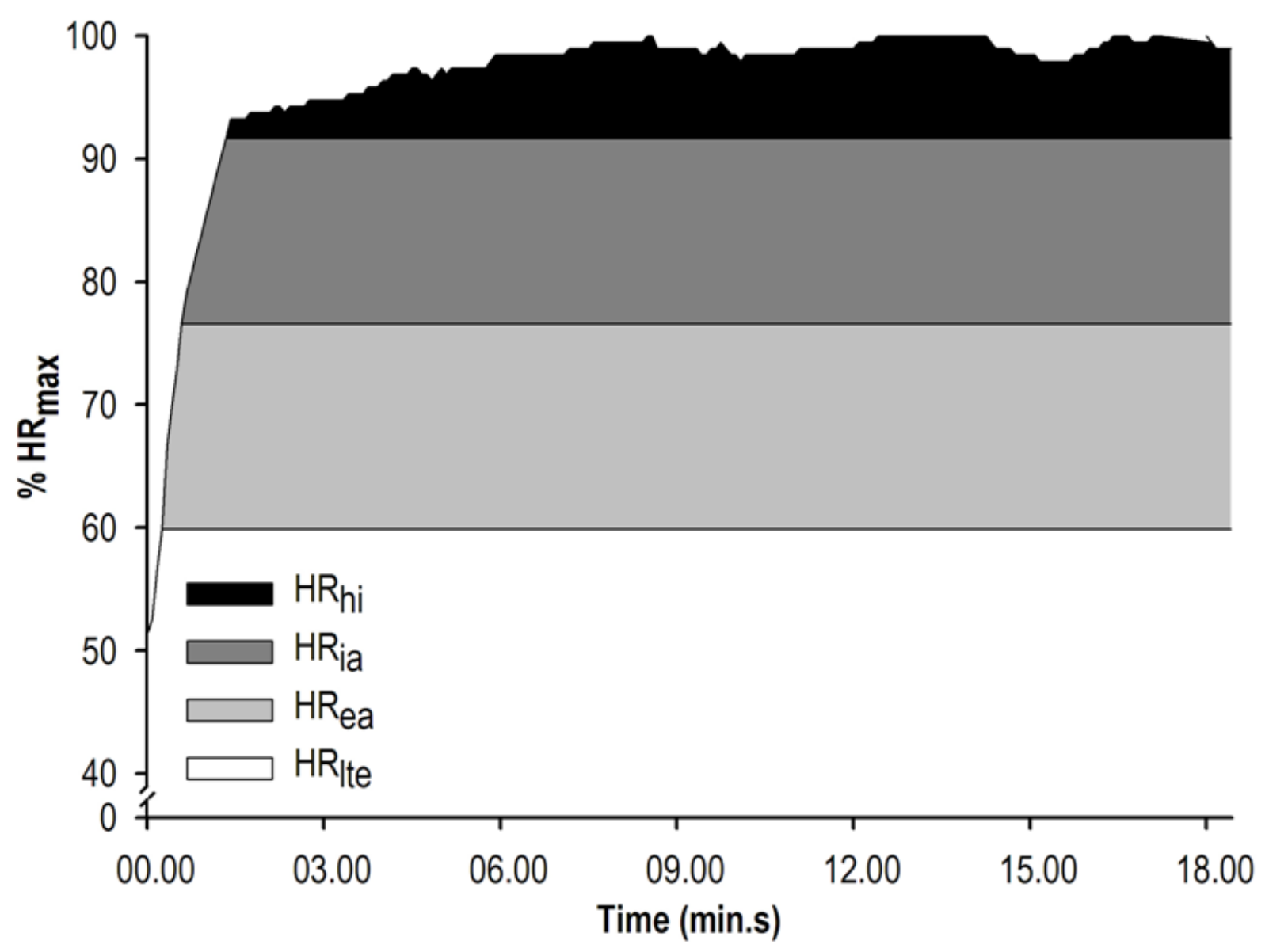

Figure 1. Athlete' s heart rate profile (expressed as mean $\%$ of $\mathrm{HR}_{\max }$ ) during the $5-\mathrm{km}$ indoor RW. HRlte, long term endurance range $\left(<117 \mathrm{~b} \cdot \mathrm{min}^{-1}\right)$; HRea, extensive aerobic range $\left(117-147 \mathrm{~b} \cdot \mathrm{min}^{-1}\right)$; HRia, intensive aerobic range $\left(147-177 \mathrm{~b} \cdot \mathrm{min}^{-1}\right)$; HRhi, high intensity range $\left(>177 \mathrm{~b} \cdot \mathrm{min}^{-1}\right)$. 
Figure 2

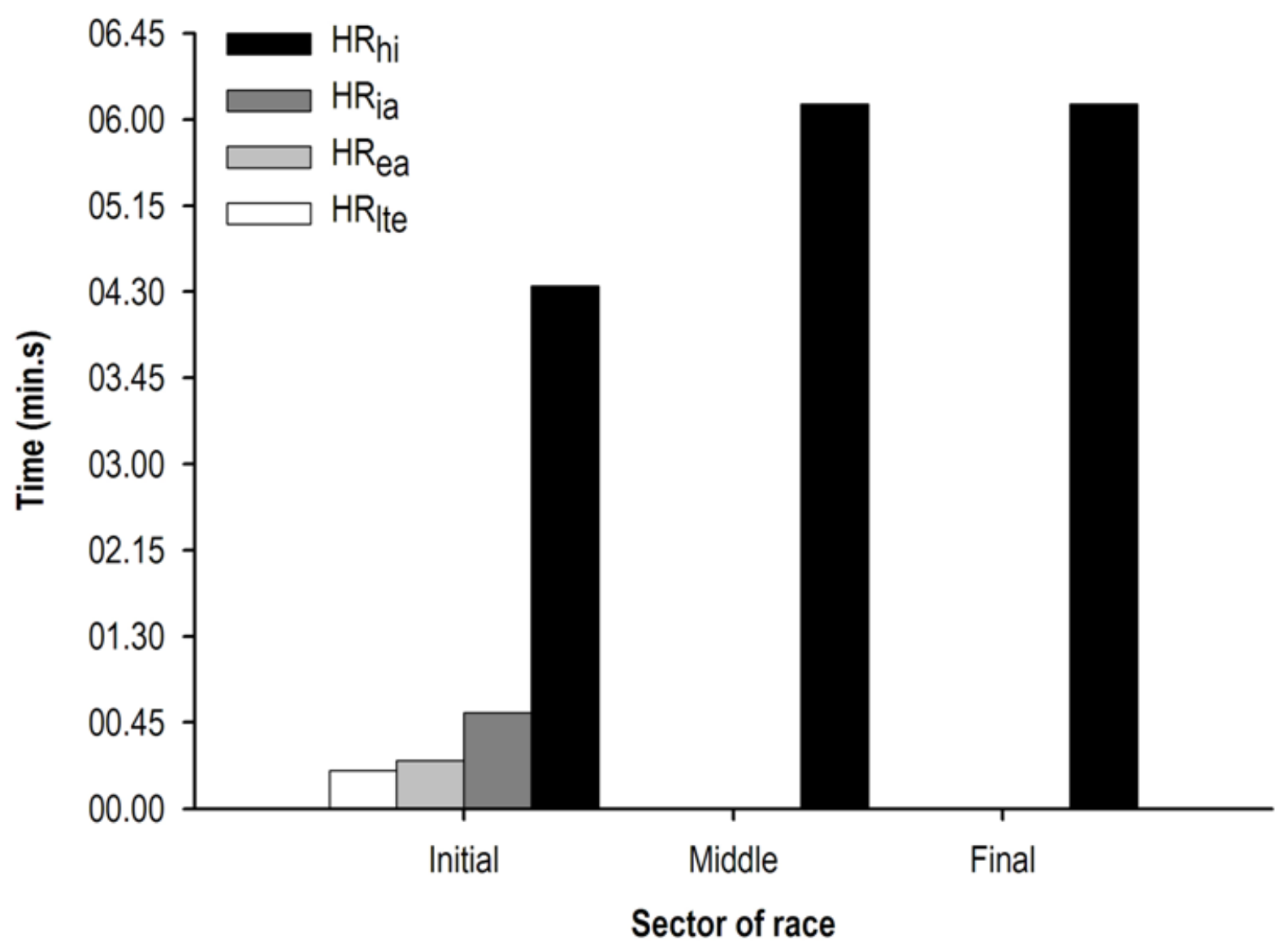

Figure 2. Time spent at each HR range in comparison to the sector of the race. HRlte, long term endurance range $\left(<117 \mathrm{~b} \cdot \mathrm{min}^{-1}\right)$; HRea, extensive aerobic range $\left(117-147 \mathrm{~b} \cdot \mathrm{min}^{-1}\right)$; HRia, intensive aerobic range $\left(147-177 \mathrm{~b} \cdot \mathrm{min}^{-1}\right)$; HRhi, high intensity range $\left(>177 \mathrm{~b} \cdot \mathrm{min}^{-1}\right)$. 
Figure 3

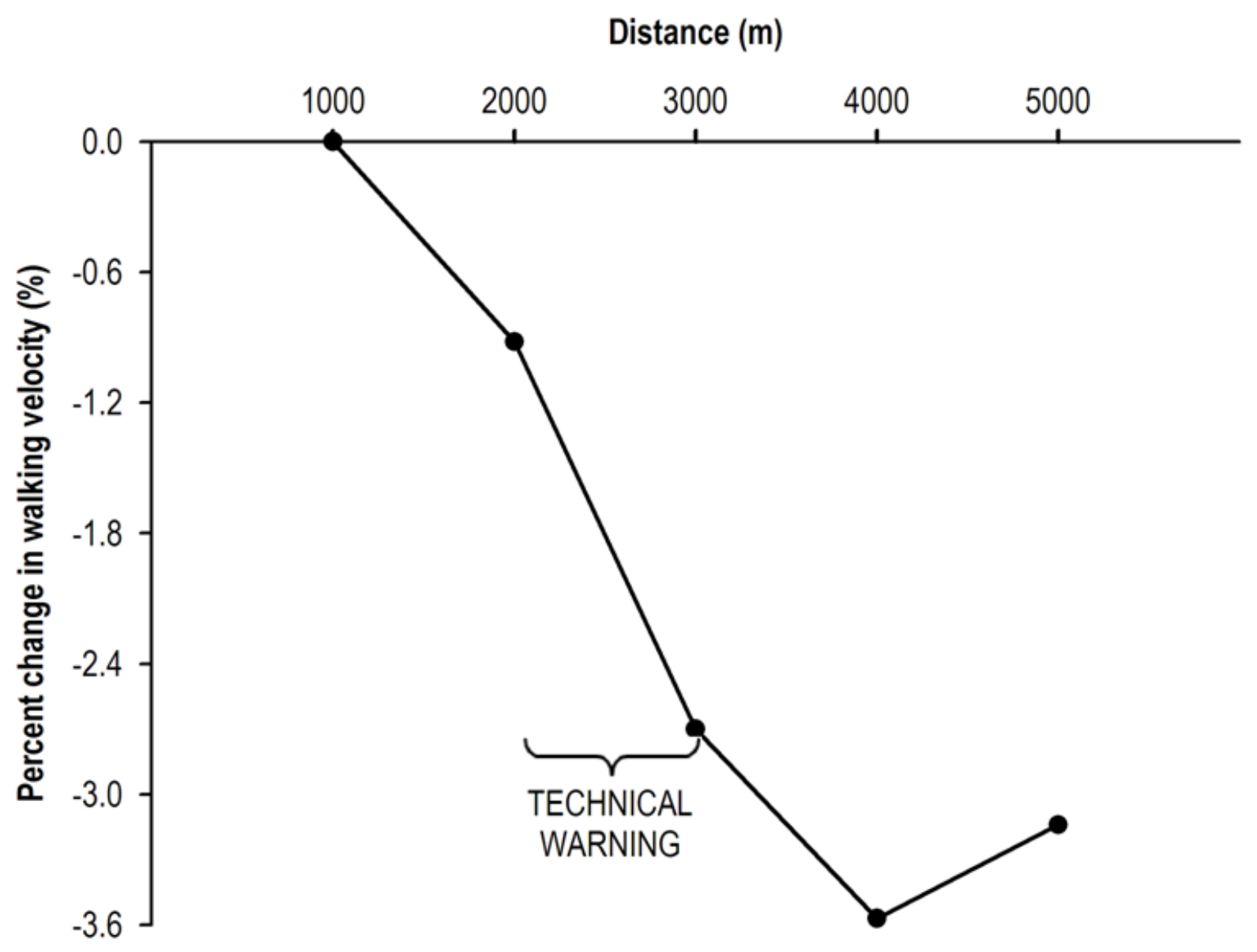

Figure 3. A plot of the athlete' s walking velocity (expressed as \% change compared to the first split-time) during the race in relation to the distance $(1000-\mathrm{m})$ completed. Curly bracket represents the distance point where the athlete received the technical warning. 


\section{BIBLIOGRAFIA}

Abbiss, C. R. \& Laursen, P. B. (2008). Describing and understanding pacing strategies during athletic competition. Sports Med, 38, 239-252.

Abbiss, C. R. \& Laursen, P. B. (2005). Models to explain fatigue during prolonged endurance cycling. Sports Med, 35, 865-898.

Achten, J. \& Jeukendrup, A. E. (2003). Heart rate monitoring: applications and limitations. Sports Med, 33, 517-538.

Alvarez-Ramirez, J. (2002). An improved Peronnet-Thibault mathematical model of human running performance. Eur J Appl Physiol, 86, 517-525.

Arsac, L. M. \& Locatelli, E. (2002). Modeling the energetics of 100-m running by using speed curves of world champions. J Appl Physiol, 92, 1781-1788.

Åstrand, P. \& Rodahl, K. (1986). Textbook of work physiology: Physiological bases of exercise. New York: McGraw-Hill Company.

Atkinson, G. \& Brunskill, A. (2000). Pacing strategies during a cycling time trial with simulated headwinds and tailwinds. Ergonomics, 43, 1449-1460.

Atkinson, G., Davison, R., Jeukendrup, A. \& Passfield, L. (2003). Science and cycling: current knowledge and future directions for research. J Sports Sci, 21, 767-787.

Atkinson, G., Peacock, O. \& Law, M. (2007). Acceptability of power variation during a simulated hilly time trial. Int J Sports Med, 28, 157-163.

Baldwin, J., Snow, R. J. \& Febbraio, M. A. (2000). Effect of training status and relative exercise intensity on physiological responses in men. Med Sci Sports Exerc, 32, 1648-1654.

Banister, E. (1991). Modeling elite athletic performance. In H. Green, J. McDougal \& H. Wenger (Eds.), Physiological Testing of Elite Atbletes, (pp. 403-424). Champain: Human Kinetics.

Barcroft, J. \& Margaria, R. (1932). Some effects of carbonic acid in high concentration on respiration. J Physiol, 74, 156-162.

Bassett, D. R., Jr. \& Howley, E. T. (2000). Limiting factors for maximum oxygen uptake and determinants of endurance performance. Med Sci Sports Exerc, 32, 70-84.

Bishop, D., Bonetti, D. \& Dawson, B. (2002). The influence of pacing strategy on VO2 and supramaximal kayak performance. Med Sci Sports Exerc, 34, 1041-1047.

Booth, F. W. \& Thomason, D. B. (1991). Molecular and cellular adaptation of muscle in response to exercise: perspectives of various models. Physiol Rev, 71, 541-585.

Borg, E. \& Kaijser, L. (2006). A comparison between three rating scales for perceived exertion and two different work tests. Scand J Med Sci Sports, 16, 57-69. 
Borg, G. (1970). Perceived exertion as an indicator of somatic stress. Scand J Rehabil Med, 2, 92-98.

Borg, G. A. (1982). Psychophysical bases of perceived exertion. Med Sci Sports Exerc, 14, 377-381.

Borresen, J. \& Lambert, M. I. (2009). The quantification of training load, the training response and the effect on performance. Sports Med, 39, 779-795.

Borresen, J. \& Lambert, M. I. (2008). Quantifying training load: a comparison of subjective and objective methods. Int J Sports Physiol Perform, 3, 16-30.

Bramble, D. M. \& Lieberman, D. E. (2004). Endurance running and the evolution of Homo. Nature, 432, 345-352.

Brisswalter, J., Fougeron, B. \& Legros, P. (1998). Variability in energy cost and walking gait during race walking in competitive race walkers. Med Sci Sports Exerc, 30, 1451-1455.

Cairns, M. A., Burdett, R. G., Pisciotta, J. C. \& Simon, S. R. (1986). A biomechanical analysis of racewalking gait. Med Sci Sports Exerc, 18, 446-453.

Cairns, S. P. (2006). Lactic acid and exercise performance : culprit or friend? Sports Med, 36, 279-291.

Cavagna, G. A. \& Franzetti, P. (1981). Mechanics of competition walking. J Physiol, 315, 243-251.

Cavagna, G. A., Thys, H. \& Zamboni, A. (1976). The sources of external work in level walking and running. J Physiol, 262, 639-657.

Cavanagh, P. R. \& Williams, K. R. (1982). The effect of stride length variation on oxygen uptake during distance running. Med Sci Sports Exerc, 14, 30-35.

Christiansen, J., Douglas, C. G. \& Haldane, J. S. (1914). The absorption and dissociation of carbon dioxide by human blood. J Physiol, 48, 244-271.

Coetzer, P., Noakes, T. D., Sanders, B., Lambert, M. I., Bosch, A. N., Wiggins, T. et al. (1993). Superior fatigue resistance of elite black South African distance runners. J Appl Physiol, 75, 1822-1827.

Coutts, A. J., Rampinini, E., Marcora, S. M., Castagna, C. \& Impellizzeri, F. M. (2009). Heart rate and blood lactate correlates of perceived exertion during small-sided soccer games. J Sci Med Sport, 12, 79-84.

Coyle, E. F. \& Gonzalez-Alonso, J. (2001). Cardiovascular drift during prolonged exercise: new perspectives. Exerc Sport Sci Rev, 29, 88-92.

Crompton, R. H., Vereecke, E. E. \& Thorpe, S. K. (2008). Locomotion and posture from the common hominoid ancestor to fully modern hominins, with special reference to the last common panin/hominin ancestor. J Anat, 212, 501-543.

Day, M. L., McGuigan, M. R., Brice, G. \& Foster, C. (2004). Monitoring exercise intensity during resistance training using the session RPE scale. J Strength Cond Res, 18, 353-358.

de Koning, J. J., Bobbert, M. F. \& Foster, C. (1999). Determination of optimal pacing strategy in track cycling with an energy flow model. J Sci Med Sport, 2, 266-277. 
Edwards, S. (1993). High performance training and racing. In S. Edwards (Ed.), The Heart Rate Monitor Book, (pp. 113-123). Sacramento: Feet Fleet Press.

Elftman, H. (1951). The basic pattern of human locomotion. Ann N Y Acad Sci, 51, 1207-1212.

Elftman, H. (1938). The Measurement of the External Force in Walking. Science, 88, 152-153.

Elftman, H. \& Manter, J. (1935). The Evolution of the Human Foot, with Especial Reference to the Joints. J Anat, 70, 56-67.

Esteve-Lanao, J., San Juan, A. F., Earnest, C. P., Foster, C. \& Lucia, A. (2005). How do endurance runners actually train? Relationship with competition performance. Med Sci Sports Exerc, 37, 496-504.

Farley, G. R. \& Hamley, E. J. (1978). Progressive changes in energy cost during a three-hour racewalk exercise. BrJ Sports Med, 12, 176-178.

Fleagle, J. G. (1976). Locomotion and posture of the Malayan siamang and implications for hominoid evolution. Folia Primatol (Basel), 26, 245-269.

Fleagle, J. G. (1979). Primate adaptation and evolution. New York: Academic Press.

Foster, C. (1998). Monitoring training in athletes with reference to overtraining syndrome. Med Sci Sports Exerc, 30, 1164-1168.

Foster, C., Daines, E., Hector, L., Snyder, A. C. \& Welsh, R. (1996). Athletic performance in relation to training load. Wis Med J, 95, 370-374.

Foster, C., deKoning, J. J., Hettinga, F., Lampen, J., Dodge, C., Bobbert, M. et al. (2004). Effect of competitive distance on energy expenditure during simulated competition. Int J Sports Med, 25, 198-204.

Foster, C., Florhaug, J. A., Franklin, J., Gottschall, L., Hrovatin, L. A., Parker, S. et al. (2001). A new approach to monitoring exercise training. J Strength Cond Res, 15, 109-115.

Foster, C., Green, M. A., Snyder, A. C. \& Thompson, N. N. (1993). Physiological responses during simulated competition. Med Sci Sports Exerc, 25, 877-882.

Foster, C., Hector, L. L., Welsh, R., Schrager, M., Green, M. A. \& Snyder, A. C. (1995). Effects of specific versus cross-training on running performance. Eur J Appl Physiol Occup Physiol, 70, 367-372.

Foster, C., Hoyos, J., Earnest, C. \& Lucia, A. (2005). Regulation of energy expenditure during prolonged athletic competition. Med Sci Sports Exerc, 37, 670-675.

Foster, C., Schrager, M., Snyder, A. C. \& Thompson, N. N. (1994). Pacing strategy and athletic performance. Sports Med, 17, 77-85.

Fritzsche, R. G., Switzer, T. W., Hodgkinson, B. J. \& Coyle, E. F. (1999). Stroke volume decline during prolonged exercise is influenced by the increase in heart rate. J Appl Physiol, 86, 799805 .

Garland, S. W. (2005). An analysis of the pacing strategy adopted by elite competitors in $2000 \mathrm{~m}$ rowing. BrJ Sports Med, 39, 39-42. 
Gilman, M. B. (1996). The use of heart rate to monitor the intensity of endurance training. Sports Med, 21, 73-79.

Gilman, M. B. \& Wells, C. L. (1993). The use of heart rates to monitor exercise intensity in relation to metabolic variables. Int J Sports Med, 14, 339-344.

Green, D. J., Watts, K., Maiorana, A. J. \& O'Driscoll, J. G. (2001). A comparison of ambulatory oxygen consumption during circuit training and aerobic exercise in patients with chronic heart failure. J Cardiopulm Rehabil, 21, 167-174.

Green, H. J. (1997). Mechanisms of muscle fatigue in intense exercise. J Sports Sci, 15, 247-256.

Groslambert, A. \& Mahon, A. D. (2006). Perceived exertion : influence of age and cognitive development. Sports Med, 36, 911-928.

Hagberg, J. M. \& Coyle, E. F. (1983). Physiological determinants of endurance performance as studied in competitive racewalkers. Med Sci Sports Exerc, 15, 287-289.

Hanson, S. L. \& Pichert, J. W. (1986). Perceived stress and diabetes control in adolescents. Health Psychol, 5, 439-452.

Hopkins, W. G. (1991). Quantification of training in competitive sports. Methods and applications. Sports Med, 12, 161-183.

Howley, E. T., Bassett, D. R., Jr. \& Welch, H. G. (1995). Criteria for maximal oxygen uptake: review and commentary. Med Sci Sports Exerc, 27, 1292-1301.

IAAF. (2009). Competition Rules 2010-2011. Monaco: Imprimerie Multiprint.

Impellizzeri, F. M., Rampinini, E., Coutts, A. J., Sassi, A. \& Marcora, S. M. (2004). Use of RPEbased training load in soccer. Med Sci Sports Exerc, 36, 1042-1047.

Impellizzeri, F. M., Rampinini, E. \& Marcora, S. M. (2005). Physiological assessment of aerobic training in soccer. J Sports Sci, 23, 583-592.

Karvonen, M. J., Kentala, E. \& Mustala, O. (1957). The effects of training on heart rate; a longitudinal study. Ann Med Exp Biol Fenn, 35, 307-315.

Kennedy, M. D. \& Bell, G. J. (2003). Development of race profiles for the performance of a simulated 2000-m rowing race. Can J Appl Physiol, 28, 536-546.

Kimura, T. (1996). Centre of gravity of the body during the ontogeny of chimpanzee bipedal walking. Folia Primatol (Basel), 66, 126-136.

Kohrt, W. M., Morgan, D. W., Bates, B. \& Skinner, J. S. (1987). Physiological responses of triathletes to maximal swimming, cycling, and running. Med Sci Sports Exerc, 19, 51-55.

Lafrenz, A. J., Wingo, J. E., Ganio, M. S. \& Cureton, K. J. (2008). Effect of ambient temperature on cardiovascular drift and maximal oxygen uptake. Med Sci Sports Exerc, 40, 1065-1071.

Lambert, M. I., Mbamba, Z. H. \& St Clair Gibson, A. (1998). Heart rate during training and competition for long-distance running. J Sports Sci, 16, S85-90. 
Lamberts, R. P. \& Lambert, M. I. (2009). Day-to-day variation in heart rate at different levels of submaximal exertion: implications for monitoring training. J Strength Cond Res, 23, 1005-1010.

Lamberts, R. P., Lemmink, K. A., Durandt, J. J. \& Lambert, M. I. (2004). Variation in heart rate during submaximal exercise: implications for monitoring training. I Strength Cond Res, 18, 641-645.

Latham, P. W. (1908). On the Formation of Lactic Acid and Carbonic Acid during Muscular Contraction and Rigor Mortis. Biochem J, 3, 193-206.

Leakey, M. G., Feibel, C. S., McDougall, I. \& Walker, A. (1995). New four-million-year-old hominid species from Kanapoi and Allia Bay, Kenya. Nature, 376, 565-571.

Lee, C. R. \& Farley, C. T. (1998). Determinants of the center of mass trajectory in human walking and running. J Exp Biol, 201, 2935-2944.

Liedl, M. A., Swain, D. P. \& Branch, J. D. (1999). Physiological effects of constant versus variable power during endurance cycling. Med Sci Sports Exerc, 31, 1472-1477.

Lovejoy, C. O. (1988). Evolution of human walking. Sci Am, 259, 118-125.

Lucia, A., Hoyos, J., Santalla, A., Earnest, C. \& Chicharro, J. L. (2003). Tour de France versus Vuelta a Espana: which is harder? Med Sci Sports Exerc, 35, 872-878.

Mahood, N. V., Kenefick, R. W., Kertzer, R. \& Quinn, T. J. (2001). Physiological determinants of cross-country ski racing performance. Med Sci Sports Exerc, 33, 1379-1384.

Marcora, S. (2009a). Perception of effort during exercise is independent of afferent feedback from skeletal muscles, heart, and lungs. J Appl Physiol, 106, 2060-2062.

Marcora, S. M. (2008). Do we really need a central governor to explain brain regulation of exercise performance? Eur J Appl Physiol, 104, 929-931; author reply 933-925.

Marcora, S. M. (2009b). Vo2max and exercise performance. J Appl Physiol, 106, 344.

Marino, F. E. (2004). Anticipatory regulation and avoidance of catastrophe during exercise-induced hyperthermia. Comp Biochem Physiol B Biochem Mol Biol, 139, 561-569.

Mattern, C. O., Kenefick, R. W., Kertzer, R. \& Quinn, T. J. (2001). Impact of starting strategy on cycling performance. Int J Sports Med, 22, 350-355.

Menier, D. R. \& Pugh, L. G. (1968). The relation of oxygen intake and velocity of walking and running, in competition walkers. J Physiol, 197, 717-721.

Midgley, A. W., McNaughton, L. R. \& Jones, A. M. (2007). Training to enhance the physiological determinants of long-distance running performance: can valid recommendations be given to runners and coaches based on current scientific knowledge? Sports Med, 37, 857-880.

Minetti, A. E., Capelli, C., Zamparo, P., di Prampero, P. E. \& Saibene, F. (1995). Effects of stride frequency on mechanical power and energy expenditure of walking. Med Sci Sports Exerc, 27, 1194-1202. 
Morgan, D. W. \& Martin, P. E. (1986). Effects of stride length alteration on racewalking economy. Can J Appl Sport Sci, 11, 211-217.

Morton, R. H., Fitz-Clarke, J. R. \& Banister, E. W. (1990). Modeling human performance in running. J Appl Pbysiol, 69, 1171-1177.

Murray, M. P., Guten, G. N., Mollinger, L. A. \& Gardner, G. M. (1983). Kinematic and electromyographic patterns of Olympic race walkers. Am J Sports Med, 11, 68-74.

Noble, R. J. \& Robertson, R. J. (1996). Perceived exertion. Champaign: Human Kinetics.

Oosthuyse, T. \& Carter, R. N. (1999). Plasma lactate decline during passive recovery from highintensity exercise. Med Sci Sports Exerc, 31, 670-674.

Owles, W. H. (1930). Alterations in the lactic acid content of the blood as a result of light exercise, and associated changes in the co(2)-combining power of the blood and in the alveolar co(2) pressure. J Physiol, 69, 214-237.

Padilla, S., Mujika, I., Angulo, F. \& Goiriena, J. J. (2000). Scientific approach to the 1-h cycling world record: a case study. J Appl Physiol, 89, 1522-1527.

Reilly, T. (1997). Energetics of high-intensity exercise (soccer) with particular reference to fatigue. $J$ Sports Sci, 15, 257-263.

Reilly, T., Hopkins, J. \& Howlett, N. (1979). Fitness test profiles and trainng intensities in skilled race-walkers. Br J Sports Med, 13, 70-76.

Richmond, B. G., Begun, D. R. \& Strait, D. S. (2001). Origin of human bipedalism: The knucklewalking hypothesis revisited. Am J Phys Anthropol, Suppl 33, 70-105.

Robinson, D. M., Robinson, S. M., Hume, P. A. \& Hopkins, W. G. (1991). Training intensity of elite male distance runners. Med Sci Sports Exerc, 23, 1078-1082.

Robinson, S., Robinson, D. L., Mountjoy, R. J. \& Bullard, R. W. (1958). Influence of fatigue on the efficiency of men during exhausting runs. J Appl Physiol, 12, 197-201.

Rowell, L. B. (1974). Human cardiovascular adjustments to exercise and thermal stress. Physiol Rev, 54, 75-159.

Sandals, L. E., Wood, D. M., Draper, S. B. \& James, D. V. (2006). Influence of pacing strategy on oxygen uptake during treadmill middle-distance running. Int J Sports Med, 27, 37-42.

Schmitt, D. (2003a). Evolutionary implications of the unusual walking mechanics of the common marmoset (C. jacchus). Am J Phys Anthropol, 122, 28-37.

Schmitt, D. (2003b). Insights into the evolution of human bipedalism from experimental studies of humans and other primates. J Exp Biol, 206, 1437-1448.

Senut, B. (2002). From apes to humans: locomotion as a key feature for phylogeny. Z Morphol Anthropol, 83, 351-360.

Siff, M. C. \& Verkhoshansky, Y. V. (1999). Supertraining. Denver: Supertraining Internationa. 
Skinner, J. S., Wilmore, K. M., Krasnoff, J. B., Jaskolski, A., Jaskolska, A., Gagnon, J. et al. (2000). Adaptation to a standardized training program and changes in fitness in a large, heterogeneous population: the HERITAGE Family Study. Med Sci Sports Exerc, 32, 157-161.

Smith, D. J. (2003). A framework for understanding the training process leading to elite performance. Sports Med, 33, 1103-1126.

Stagno, K. M., Thatcher, R. \& van Someren, K. A. (2007). A modified TRIMP to quantify the inseason training load of team sport players. J Sports Sci, 25, 629-634.

Stern, J. T., Jr. \& Susman, R. L. (1983). The locomotor anatomy of Australopithecus afarensis. Am J Phys Anthropol, 60, 279-317.

Susman, R. L. \& Stern, J. T. (1982). Functional Morphology of Homo habilis. Science, 217, 931-934.

Swain, D. P. (1997). A model for optimizing cycling performance by varying power on hills and in wind. Med Sci Sports Exerc, 29, 1104-1108.

Swain, D. P. \& Leutholtz, B. C. (1997). Heart rate reserve is equivalent to \%VO2 reserve, not to \%VO2max. Med Sci Sports Exerc, 29, 410-414.

Swain, D. P., Leutholtz, B. C., King, M. E., Haas, L. A. \& Branch, J. D. (1998). Relationship between \% heart rate reserve and \% VO2 reserve in treadmill exercise. Med Sci Sports Exerc, 30, 318-321.

Sweet, T. W., Foster, C., McGuigan, M. R. \& Brice, G. (2004). Quantitation of resistance training using the session rating of perceived exertion method. J Strength Cond Res, 18, 796-802.

Thompson, K. G., Haljand, R. \& MacLaren, D. P. (2000). An analysis of selected kinematic variables in national and elite male and female 100-m and 200-m breaststroke swimmers. J Sports Sci, $18,421-431$.

Thompson, K. G., MacLaren, D. P., Lees, A. \& Atkinson, G. (2003). The effect of even, positive and negative pacing on metabolic, kinematic and temporal variables during breaststroke swimming. Eur J Appl Physiol, 88, 438-443.

Thompson, K. G., MacLaren, D. P., Lees, A. \& Atkinson, G. (2004). The effects of changing pace on metabolism and stroke characteristics during high-speed breaststroke swimming. J Sports Sci, 22, 149-157.

Tucker, R. (2009). The anticipatory regulation of performance: the physiological basis for pacing strategies and the development of a perception-based model for exercise performance. $\mathrm{BrJ}$ Sports Med, 43, 392-400.

Tucker, R., Marle, T., Lambert, E. V. \& Noakes, T. D. (2006). The rate of heat storage mediates an anticipatory reduction in exercise intensity during cycling at a fixed rating of perceived exertion. J Physiol, 574, 905-915.

Tucker, R., Rauch, L., Harley, Y. X. \& Noakes, T. D. (2004). Impaired exercise performance in the heat is associated with an anticipatory reduction in skeletal muscle recruitment. Pflugers Arch, $448,422-430$. 
Umberger, B. R. \& Martin, P. E. (2007). Mechanical power and efficiency of level walking with different stride rates. J Exp Biol, 210, 3255-3265.

van Ingen Schenau, G. J., de Koning, J. J. \& de Groot, G. (1992). The distribution of anaerobic energy in 1000 and 4000 metre cycling bouts. Int J Sports Med, 13, 447-451.

Vernillo, G., Agnello, L., Drake, A., Padulo, J., Piacentini, M. F. \& Torre, A. L. An Observational Study on the Perceptive and Physiological Variables During a 10,000-m Race Walking Competition. J Strength Cond Res.

Vernillo, G., Piacentini, M. F., Drake, A., Agnello, L., Fiorella, P. \& La Torre, A. (2011). Exercise intensity and pacing strategy of a $5-\mathrm{km}$ indoor race walk during a World Record attempt: a case study. J Strength Cond Res, 25, 2048-2052.

Viru, A. (1995). Adaptation in sports training. Boca Raton: CRC Press.

Viru, A. \& Viru, M. (2000). Nature of training effects. In W. Garrett \& D. Kirkendall (Eds.), Exercise and Sport Science, (pp. 67-95). Philadelphia: Lippincott Williams \& Williams.

Wangenheim, M., Carlsoo, S., Nordgren, B. \& Linroth, K. (1986). Perception of efforts in working postures. Ups J Med Sci, 91, 53-66.

Ward, C. V., Leakey, M. G., Brown, B., Brown, F., Harris, J. \& Walker, A. (1999). South Turkwel: a new pliocene hominid site in Kenya. J Hum Evol, 36, 69-95.

Wilberg, R. B. \& Pratt, J. (1988). A survey of the race profiles of cyclists in the pursuit and kilo track events. Can J Sport Sci, 13, 208-213.

Wingo, J. E., Lafrenz, A. J., Ganio, M. S., Edwards, G. L. \& Cureton, K. J. (2005). Cardiovascular drift is related to reduced maximal oxygen uptake during heat stress. Med Sci Sports Exerc, 37, 248-255.

Xu, F. \& Rhodes, E. C. (1999). Oxygen uptake kinetics during exercise. Sports Med, 27, 313-327.

Yoshida, T., Udo, M., Iwai, K., Muraoka, I., Tamaki, K., Yamaguchi, T. et al. (1989). Physiological determinants of race walking performance in female race walkers. Br J Sports Med, 23, 250254.

Zarrugh, M. Y. \& Radcliffe, C. W. (1978). Predicting metabolic cost of level walking. Eur J Appl Physiol Occup Physiol, 38, 215-223.

Ziv, G. \& Rotstein, A. (2009). Physiological characteristics of the preferred transition speed in racewalkers. Med Sci Sports Exerc, 41, 797-804. 